\title{
Molecular basis of intraspecific differentiation for heavy metal tolerance in the copper moss Scopelophila cataractae
}

\author{
M. Teresa Boquete ${ }^{1}$, Marc W. Schmid ${ }^{2}$, Niels C.A.M. Wagemaker ${ }^{3}$, Sarah B. Carey ${ }^{4}$, \\ Stuart F. McDaniel ${ }^{4}$, Christina Richards ${ }^{5}$, and Conchita Alonso ${ }^{1}$ \\ ${ }^{1}$ Estacion Biologica de Donana CSIC \\ ${ }^{2}$ MWSchmid GmbH Glarus Switzerland \\ ${ }^{3}$ Faculty of Science Raboud University Nijmegen The Netherlands \\ ${ }^{4}$ University of Florida Department of Biology \\ ${ }^{5}$ University of South Florida Department of Integrative Biology
}

February 13, 2022

\begin{abstract}
Bryophytes' remarkable capacity to tolerate extreme abiotic conditions allows us to enhance our understanding of the diversity of molecular mechanisms involved in plant stress response. Here, we used next generation sequencing to study DNA methylation and gene expression changes in plants from four populations of the metallophyte moss Scopelophila cataractae experimentally exposed to either $\mathrm{Cd}$ or $\mathrm{Cu}$. These populations previously showed differences in tolerance to both metals, so here, we aimed at uncovering the molecular basis of this phenotypic differentiation. We found no evidence of genetic differentiation among the populations studied. The epigenetic data, however, showed limited but significant population-specific changes in DNA methylation in response to both metals. Exposure to acute $\mathrm{Cu}$ stress in the laboratory led to the downregulation of genes involved in heavy metal tolerance in both populations regardless of their tolerance level, but this response was quantitatively higher in the most tolerant. We propose that chronic exposure to varying levels of heavy metals in the field led to potentially non-genetically-based intraspecific differentiation for heavy metal tolerance in S. cataractae. The most tolerant plants invested more in constitutive protection and were more efficient in entering a conservative state when faced with acute $\mathrm{Cu}$ stress.
\end{abstract}

Molecular basis of intraspecific differentiation for heavy metal tolerance in the copper moss Scopelophila cataractae

M. Teresa Boquete ${ }^{1,5} 11 \mathrm{M}$. Teresa Boquete is the Corresponding author. Department of Evolutionary Ecology, Estación Biológica de Doñana-CSIC, Avenida Americo Vespucio n⿳⺈ 26, 41092, Sevilla, Spain. teresa.boquete@gmail.com, Marc W. Schmid 222contact@mwschmid.ch, Niels C.A.M. Wagemaker ${ }^{3} 33$ n.wagemaker@science.ru.nl, Sarah B. Carey ${ }^{4} 44$ scarey@hudsonalpha.org, Stuart F. McDaniel ${ }^{4} 55$ stuartmcdaniel@ufl.edu, Christina L. Richards ${ }^{5,6} 66 \mathrm{clr} @ u s f . e d u, \quad$ Conchita Alonso ${ }^{1} 77$ conalo@ebd.csic.es

${ }^{1}$ Department of Evolutionary Ecology, Estación Biológica de Doñana, CSIC, Sevilla, Spain.

${ }^{2}$ MWSchmid GmbH, Glarus, Switzerland.

${ }^{3}$ Faculty of Science, Raboud University, Nijmegen, The Netherlands.

${ }^{4}$ Department of Biology, University of Florida, Gainesville, FL, USA

${ }^{5}$ Department of Integrative Biology, University of South Florida, Tampa, FL, USA. 
${ }^{6}$ Plant Evolutionary Ecology group, University of Tübingen, Tübingen, Germany.

Funding information: This research has received funding from the European Union's Horizon 2020 research and innovation programme under the Marie Skłodowska-Curie grant agreement No 704141-BryOmics. Dr. M. Teresa Boquete is currently supported by the Juan de la Cierva-Incorporación program from the Spanish Ministry of Science, Innovation and Universities (IJC2018-035018-I)

\section{Paper structure}

Total word count: $7859 \mathrm{w}$.

Number of figures: 2

Number of tables: 2

Supplementary materials: 7 figures and 8 tables

Running Head : Molecular mechanisms of heavy metal tolerance in bryophytes.

\section{Abstract}

Bryophytes' remarkable capacity to tolerate extreme abiotic conditions allows us to enhance our understanding of the diversity of molecular mechanisms involved in plant stress response. Here, we used next generation sequencing to study DNA methylation and gene expression changes in plants from four populations of the metallophyte moss Scopelophila cataractae experimentally exposed to either $\mathrm{Cd}$ or $\mathrm{Cu}$. These populations previously showed differences in tolerance to both metals, so here, we aimed at uncovering the molecular basis of this phenotypic differentiation. We found no evidence of genetic differentiation among the populations studied. The epigenetic data, however, showed limited but significant population-specific changes in DNA methylation in response to both metals. Exposure to acute $\mathrm{Cu}$ stress in the laboratory led to the downregulation of genes involved in heavy metal tolerance in both populations regardless of their tolerance level, but this response was quantitatively higher in the most tolerant. We propose that chronic exposure to varying levels of heavy metals in the field led to potentially non-genetically-based intraspecific differentiation for heavy metal tolerance in $S$. cataractae. The most tolerant plants invested more in constitutive protection and were more efficient in entering a conservative state when faced with acute $\mathrm{Cu}$ stress.

Keywords : abiotic stress; cadmium; copper; DNA methylation; epigenetics; gene expression; heavy metals; phenotypic variation; reduced representation bisulfite sequencing; RNA sequencing.

\section{Acknowledgments}

We thank Dr. Carlos M. Herrera for his advice throughout the development of the project; we also thank Dr. Jonathan A. Shaw and Dr. Blanka Aguero for their help with field sample localization and collection, Luiza Silva Simoes and Olivia Santiago for help during laboratory work at USF, and Dr. Mariano Alvarez, Dr. Marta Robertson, Dr. Sabrina McNew, Dr. Jeannie Mounguer, and Sandra Voors for continuous discussions and feedback about the epiGBS data analyses. This research has received funding from the European Union's Horizon 2020 research and innovation programme under the Marie Skłodowska-Curie grant agreement No 704141-BryOmics. Dr. M. Teresa Boquete is currently supported by the Juan de la Cierva-Incorporación program from the Spanish Ministry of Science, Innovation and Universities (IJC2018-035018-I).

\section{Introduction}

Heavy metals are amongst the most hazardous pollutants to living organisms due to their high toxicity and persistence in the environment ${ }^{1-4}$. Multiple studies showed that heavy metals damage the cell membrane system and negatively affect plant development, growth, and reproduction ${ }^{5-8}$, generate reactive oxygen species $(\mathrm{ROS})^{9,10}$, and may alter the structure of essential plant biomolecules ${ }^{11-14}$. Still, some species have adapted 
to living in heavy metal-enriched environments, and are able to survive and accumulate concentrations of heavy metals that greatly exceed the tolerance limits of most other plants ${ }^{15-17}$.

Metal tolerant species often rely on the enhanced expression of genes related to heavy metal homeostasis - i.e. transport, chelation, and sequestration of metals - relative to closely related non-tolerant ones ${ }^{18-23}$. Thus, constitutive overexpression of heavy metal homeostasis-related genes (e.g. ABC metal transporters and enzymes involved in oxidative stress relief ${ }^{20,21}$ ) often underlies differences between tolerant and non-tolerant plants (but see ${ }^{24,25}$ for the contribution of gene copy number variation). Yet, the regulatory mechanisms determining the differences in gene expression are still poorly understood. Gene expression in eukaryotic genomes is, in part, tightly regulated by epigenetic mechanisms, the set of chromatin modifications that control chromatin structure, and thus, the accessibility of the transcriptional machinery to genes ${ }^{26,27}$. In plants, epigenetic mechanisms, such as DNA methylation, histone modifications, and small non-coding RNAs, are known to regulate gene expression changes in response to developmental and environmental cues (e.g. ${ }^{28-33}$ ). Based on this evidence, epigenetic mechanisms are currently considered potentially important regulators of plants response to stress.

So far, experimental evidence on the contribution of epigenetic mechanisms to plant heavy metal tolerance has shown that DNA methylation could have a direct protective role. For example, metal tolerant plants like Arabidopsis halleri - in response to $\mathrm{Cd}$ - and Noccaea caerulescens - in response to Ni showed hypermethylation of DNA and no heavy metal-induced DNA damage ${ }^{34,35}$. Non-tolerant species like the moss Physcomitrium patens, however, showed overall DNA hypomethylation and significant signs of DNA strand breaks in response to $\mathrm{Mn}^{36}$. Studies employing anonymous DNA methylation markers or immunolabelling techniques reported DNA hypermethylation in response to exposure to high Al levels in wheat (Triticum aestivum $)^{37}$ and maize ${ }^{38}$, to $\mathrm{Pb}$ in maize (Zea mays) ${ }^{39}$, to $\mathrm{Cd}$ in Posidonia oceanica ${ }^{40}$ and Nicotiana benthamiana $^{41}$, and to $\mathrm{Cd}, \mathrm{Ni}$ and $\mathrm{Cr}$ in Trifolium repensand Cannabis sativa ${ }^{42}$. In contrast, DNA hypomethylation was reported in response to $\mathrm{Cd}$ in rapeseed (Brassica napus) ${ }^{43}$ and the red algaGracilaria dura ${ }^{44}$, to $\mathrm{Al}$ in triticale (xTriticosecale ) ${ }^{45,46}$, to $\mathrm{Cu}$ and $\mathrm{Zn}$ inPopulus alba ${ }^{47}$, and to low $\mathrm{Al}$ levels in wheat ${ }^{37}$. Epigenetic mechanisms like DNA methylation can thus be modified in response to heavy metal exposure.

More targeted studies have demonstrated that epigenetic changes induced by heavy metals can lead to changes in the expression of genes involved in heavy metal tolerance. For example, work with methylation mutants confirmed that CG and H3K9me2 hypomethylation upstream of the metal responsive metallochaperone, $O s$ $H M P$, led to its overexpression and enhanced $\mathrm{Cd}$ tolerance in rice (Oryza sativa $)^{48}$. Similar DNA and histone hypomethylation led to increased expression of a $\mathrm{Cd}$ tolerance factor (OsCTF ) that enhanced $\mathrm{Cd}$ tolerance in rice ${ }^{49}$. Other studies used transformation of Arabidopsis thaliana with an S-adenosylmethionine synthetase (SAMS) gene, which encodes an enzyme that catalyzes the biosynthesis of SAM, the main methyl group donor for DNA methylation in plants. This transformation conferred $\mathrm{Al}$ as well as $\mathrm{Cd}, \mathrm{Cu}$, and $\mathrm{Zn}$ tolerance to this species ${ }^{50}$. The overexpression of specific heavy metal detoxification transporters in a $\mathrm{Pb}$, $\mathrm{Cd}$, and $\mathrm{Zn}$ tolerant variety of wheat has been linked to $\mathrm{CG}$ hypomethylation in their promoter region in response to all three metals ${ }^{51}$. Prolonged Cr exposure during selection of a Cr-tolerant strain of the green alga Scenedesmus acutus led to new DNA methylation and expression patterns in protein-coding genes involved in $\mathrm{Cr}$ tolerance. MicroRNAs also seem to contribute to plant response to $\mathrm{Cr}$ stress in rice ${ }^{52}$, $\mathrm{Cd}$ in rice $^{53,54}$ and rapeseed ${ }^{55,56}$, and $\mathrm{Al}, \mathrm{Cd}$, and $\mathrm{Hg}$ in Medicago truncatula ${ }^{57}$. Finally, some studies showed that DNA methylation changes induced during heavy metal exposure can be transmitted to the offspring in rice $^{58,59}$ and Arabidopsis ${ }^{60}$ potentially leading to heritable changes in gene expression patterns. Collectively, these data suggest that epigenetic regulation could play an important role in the plant response to heavy metal stress. However, these studies are based on a phylogenetically restricted set of species, largely from the Brassicaceae and Poaceae (i.e. crops and model plants like Arabidopsis ), which may not be representative of plants as a whole.

Bryophytes, the sister group to the vascular plants, have long been known for their capacity to tolerate high concentrations of heavy metals in their tissues ${ }^{61}$. Most of what is presently known about their tolerance mechanisms comes from biochemical and physiological studies. Mechanisms such as retention of heavy me- 
tals in extracellular wax-like substances ${ }^{62}$, sequestration in the cell wall ${ }^{63-66}$, preferential accumulation in specific parts/organs of the leafy plant - i.e. gametophore ${ }^{67-69}$, or the activation of the ROS scavenging machinery and induction of metal-chelating proteins ${ }^{70}$, have been reported to mitigate heavy metal stress in bryophytes. However, we lack data concerning the molecular underpinnings of these anatomical or physiological phenomena. Filling this gap will enable us to test whether the land plants employ conserved pathways to tolerate heavy metal stress, or whether each lineage has evolved novel mechanisms.

In this study, we used a reduced representation bisulfite sequencing technique (hereafter epiGBS ${ }^{71}$ ), and RNA sequencing (RNAseq) to quantify the effect of $\mathrm{Cd}$ and $\mathrm{Cu}$ exposure on DNA methylation and gene expression in the copper moss Scopelophila cataractae(Mitt.) Broth, a species with high affinity for heavy metal-, especially $\mathrm{Cu}$-enriched substrates ${ }^{72}$. We generated laboratory cultures, in control or test (either $\mathrm{Cd}$ or $\mathrm{Cu}$ ) conditions, of samples from four field microsites (maximum distance between microsites $<500$ m) within a former copper mine. Using this experimental set up, we previously found that phenotypic differences for $\mathrm{Cd}$ and $\mathrm{Cu}$ tolerance (defined as the ability to maintain vegetative growth in metal stressed vs. control conditions, sensu ${ }^{73}$ ) in this species were maintained in the laboratory ${ }^{68}$. The more tolerant plants were found in the center of the mine growing in microhabitats devoid of any vegetation and exposed to higher metal concentrations, i.e., harsher environmental conditions, while less tolerant plants were from the mine edges, located in milder, less contaminated microhabitats. Here we used epigenetic and transcriptomic analyses to identify the mechanisms driving intraspecific differentiation for heavy metal tolerance in $S$. cataractae. Specifically, we addressed the following questions: (1) What are mechanisms driving intraspecific differentiation for heavy metal tolerance in S. cataractae ? (2) Does heavy metal exposure affect DNA methylation? (3) What are the main functional changes in $S$. cataractae in response to heavy metal exposure? (4) Is there any association between methylation and expression changes? Our results provided evidence for non-genetically-based intraspecific phenotypic differentiation for heavy metal tolerance in this species with the more tolerant plants investing more in constitutive protection mechanisms and being more efficient in entering a conservative state when faced with acute $\mathrm{Cu}$ stress.

\section{Materials and Methods}

\subsection{Field sampling.}

We collected plants from four populations of $S$. cataractae (Sc1 to Sc4) in a former copper mine in Silver Hill, North Carolina (USA) in September 2016. We use the term population to refer to physically unconnected and scattered patches of this species (separated by mostly bare soil), even though the distance between these patches was very short $(\sim 20$ to $\sim 300 \mathrm{~m})$. These populations were exposed to different concentrations of heavy metals in the field: the two growing in the center of the mine $(\mathrm{Sc} 2, \mathrm{Sc} 3)$ were exposed to higher concentrations of $\mathrm{Cd}$ and $\mathrm{Cu}$ than the two growing in microhabitats located at the edges of the mine (Sc1, Sc4) ${ }^{68}$. At every site, we sampled gametophytic tissue of $S$. cataractae from several moss patches.

\subsection{Common garden experiments}

We performed two common garden experiments to test separately the effect of $\mathrm{Cd}$ and $\mathrm{Cu}$ on DNA methylation and gene expression in $S$. cataractae. First, we clonally propagated each population in growth chambers at $22{ }^{\circ} \mathrm{C}$ and $16 \mathrm{~h}$ light $/ 8 \mathrm{~h}$ dark for several months (conditions maintained for all experiments); for this, we cleaned individual gametophores with deionized (DI) water and a brush under the dissection microscope, cut them into small pieces with a razor blade, and spread them into $4 \times 4 \mathrm{~cm}$ pots containing a 2:1 mixture of clay (Turface) and commercial soil. Second, we selected 50-70 gametophores from each lab grown culture, cleaned them with DI water as explained above, cut them into small pieces, and spread them in $4 \mathrm{x} 4 \mathrm{~cm}$ pots containing a previously autoclaved 2:1 mixture of clay and potting soil. We grew five replicates per population $(\mathrm{n}=4)$ and treatment $(\mathrm{n}=3)$ combination $(\mathrm{n}=60$ pots in total $)$ for 3 months in a growth chamber watering the pots with DI water every two days. After this period, we applied the treatments by watering the plants every two days with $20 \mathrm{~mL}$ of water (controls), or water containing $1 \mathrm{mM} \mathrm{Cu}(\mathrm{Cu})$, or $0.1 \mathrm{mM} \mathrm{Cd}(\mathrm{Cd})(\mathrm{n}=4-5$ replicates per population and treatment). After 30 days, we harvested the plants, blotted them with filter paper and made several aliquots that were immediately frozen in liquid $\mathrm{N}$ and stored 
at $-80^{\circ} \mathrm{C}$ for DNA methylation and gene expression analyses. Other aliquots of these same samples were used for the phenotypic characterization $\left(\mathrm{see}^{68}\right)$.

\subsection{DNA extraction and epiGBS library preparation}

We isolated genomic DNA (gDNA) from four S. cataractaepopulations (Sc1, Sc2, Sc3, Sc4) with each exposed to three treatments (control, Cd-treated, $\mathrm{Cu}$-treated) hereafter referred to as group ( $\mathrm{n}=12$ groups) with each group replicated in triplicate $(\mathrm{n}=36$ samples in total). We followed the cetyltrimethylammonium bromide (CTAB) DNA extraction protocol for recalcitrant plant tissues (https://www.protocols.io/view/high-qualitydna-extraction-protocol-from-recalcit-i8jchun) with small modifications (detailed protocol available in the Supplementary Methods - SMs). We checked the quality of the gDNA with the NanoDrop (Nanodrop 8000 Spectrophotometer; Thermo Scientific), and quantified its concentration using the Qubit 3.0 Fluorometric dsDNA BR assay kit (Q32851; Life Technologies). We obtained high quality gDNA for all samples except for one replicate of Sc3 in control conditions, which we did not include in the sequencing library.

We prepared the epiGBS libraries following ${ }^{71}$. We digested $400 \mathrm{ng}$ of gDNA from each sample with the restriction enzymePstI . Then, we ligated methylated, non-phosphorylated barcoded adapters to both ends of the digested fragments. We concentrated the library using the NucleoSpin Gel and PCR Clean-up Kit (12303368, Macherey-Nagel) and performed a fragment size selection with 0.8x SPRI beads (A63880, Agencourt AMPure XP Beckman coulter). We performed nick translation, bisulfite converted the DNA using the EZ Lightning methylation kit (Zymo Research), and amplified the library with the KAPA HIFI Uracil+ Hotstart Ready Mix (Roche) under the following PCR conditions: initial denaturation step at 95oC for 3 min; 20 cycles of $98 \mathrm{oC}$ for $10 \mathrm{~s}, 65 \mathrm{oC}$ for $15 \mathrm{~s}$, and $72 \mathrm{oC}$ for $15 \mathrm{~s}$; final extension of $72 \mathrm{oC}$ for $5 \mathrm{~min}$. Finally, we quantified the library using the Qubit dsDNA assay kit, pooled all samples using equimolar concentrations and assessed its quality by analyzing $1 \mu \mathrm{L}$ on a High Sensitivity DNA chip on an Agilent 2100 Bioanalyzer. The library was sequenced at Novogene (HK) Company Limited in Hong Kong on the Illumina HiSeq X-Ten System (PE-150 bp).

\subsection{RNA extraction and RNAseq library preparation}

Budget limitations precluded analyzing the full set of samples, so we measured gene expression on a subset of 12 samples including control and Cu-treated plants from one population from the center of the mine (Sc3) and one from the edges of the mine ( $\mathrm{Sc} 4)(\mathrm{n}=3$ replicates per population and treatment). Total RNA was isolated with the RNeasy Plant Mini Kit (74904, Qiagen) following the manufacturer instructions with small modifications (detailed protocol available in the $\mathrm{SMs}$ ).

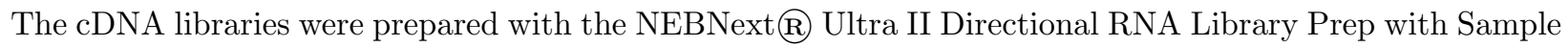
Purification Beads (E7765S, NEB) following the manufacturer's protocol with $1 \mu \mathrm{g}$ of total RNA as input material (see detailed protocol in SMs).

\subsection{Data processing}

\subsection{1 epiGBS data processing and filtering}

We used the pipeline provided by ${ }^{71}$ with a bug-fix modification (https://github.com/MWSchmid/epiGBS_Nov_2017_fixed) to process the sequencing files (sensu ${ }^{74}$ ). First, we created a de novo reference from the demultiplexed and quality trimmed sequencing reads. We mapped the reads to the de novoreference and performed strand-specific nucleotide (single nucleotide polymorphisms, SNPs) and methylation (single methylation polymorphisms, SMPs) variant calling. We filtered the resulting SNP and SMP files as follows: i) we initially removed SNPs and SMPs without a minimum coverage of 3 (i.e. 3 sequencing reads mapping to each locus) and a maximum coverage equal to the $99^{\text {th }}$ percentile of the read coverage distribution, in at least one replicate sample per group ii) we removed samples lacking more than $20 \%$ of the SNPs or more than $25 \%$ of the SMPs; and iii) after removing these samples from the experimental design, we used the original SNP and SMP dataset and removed SNPs and SMPs without a minimum coverage of 10 and a maximum coverage equal to the $99^{\text {th }}$ percentile of the read coverage distribution in at least one replicate sample per group. 
The unfiltered datasets resulting from the epiGBS pipeline consisted of 279,103 SNPs and 22.3 million SMPs across all 35 samples. The first and less strict filtering step resulted in 58,773 SNPs and 290,547 SMPs. During the second step we removed 5 samples with low SNP and SMP coverage which, in general, had a low number of sequencing reads after demultiplexing and quality trimming (Table S1). The last and stricter filtering resulted in 52,513 SNPs and 239,728 SMPs across 30 samples comprising 2-3 replicates per group (Table S1).

We created a final SMP working dataset by removing: i) SMPs with any missing values across all 30 samples to obtain a complete SMP matrix (we discarded 196,039 SMPs); ii) SMPs called on the same cytosine as a SNP (we removed 324 positions); and iii) monomorphic SMPs, i.e. SMPs with a methylation frequency [?] $5 \%$ and [?] $95 \%$ across $95 \%$ (i.e. $\mathrm{n}=28$ ) of the samples. We calculated the methylation level at each SMP in each individual sample as the number of reads mapping to one position with methylation divided by the total number of reads mapping to that position. The final matrix, containing only polymorphic SMPs, consisted of 3,769 cytosines across 30 samples. Similarly, we removed SNPs with any missing values across all 30 samples; the final SNP working dataset consisted of 23,252 SNPs.

\subsubsection{RNA-seq data processing, de novo transcriptome assembly, and transcript abundance estimation}

We quality trimmed the raw sequencing reads by removing adaptors and low-quality bases with Trimmomatic v $0.36{ }^{75}$ with a 4-base sliding window and quality threshold of 25 . These reads were used for de novo transcriptome reconstruction with Trinity v $2.8 .4^{76}$, following the protocol by ${ }^{77}$. Then, we clustered highly redundant transcripts, i.e. transcripts with $>95 \%$ sequence similarity, using CD-HIT v 4.6.6 ${ }^{78}$, and selected the longest isoform per gene using Trinity's custom script (get_longest_isoform_seq_per_trinity_gene.pl). The quality of the assembly was assessed by mapping the trimmed reads back to the assembly using Bowtie $2 \mathrm{v}$ 2.2.9 ${ }^{79}$ and its completeness by searching for orthologues with BUSCO v 3.1.0 ${ }^{80}$ using the viridiplantaeodb10 database as a reference (E-value cutoff for the blast alignments: $1 \mathrm{e}^{-06}$ ).

We estimated transcript abundance within each individual sample using RSEM ${ }^{81}$ wrapped by scripts included in Trinity (align_and_estimate_abundance.pl). This software first aligns the sequenced reads back to the transcriptome, and then provides read counts and normalized expression values for each transcript in each sample. Finally, we created a count matrix with read counts across all samples.

\subsubsection{Annotation of de novo transcriptome assembly}

We used the pipeline available within the bioinformatics platform OmicsBox ${ }^{82,83}$ to annotate the de novotranscriptome as follows: i) we performed a blast search against the non-redundant protein sequence database (nr v5) (blastx-fast; E-value cutoff: $1 \mathrm{e}^{-05}$ ); ii) we retrieved gene ontology (GO) terms for the sequences with blast hits using the gene_info and gene2accession files from the NCBI database, and UniProt IDs using the PSD, UniProt, Swiss-Prot, TrEMBL, RefSeq, GenPept and PDB databases; iii) we annotated the sequences by assigning the most reliable and specific GO terms according to their E-values $\left(<1 \mathrm{e}^{-06}\right)$ and sequence similarities (high scoring segment pair hit coverage cutoff of $80 \%$ ) as well as the quality of their annotation using the evidence code for each GO term ( 1 for experimental evidence, 0.7-0.8 for computational analysis evidence, and 0.5-0.9 for all other evidence types) ${ }^{84}$; iv) in parallel, we searched for matches between our sequences and protein domains and families within the InterPro protein databases and the EggNOG database to annotate predicted orthologues within our query sequences ${ }^{85}$; v) we merged the InterPro and EggNOG classifications with the annotation resulting from step (iii).

Additionally, we used RepeatMasker v 4.0 to annotate transposons and repeats in the de novo reference genome (obtained with the epiGBS bioinformatics pipeline) using Embryophyta as reference species collection (v.4.0.6 ${ }^{86}$ ) and DIAMOND v 0.8.22 to annotate protein coding genes with the NCBI non-redundant protein sequences database ${ }^{87}$, in order to classify epigenetic variants into different genomic features.

\subsection{Statistical analysis}

All analyses were performed in R v.3.5.1 ${ }^{88}$ running under R Studio v.1.2.5019 ${ }^{89}$. 


\subsubsection{Genetic analyses}

To determine if the phenotypic differences in tolerance were associated with genetic differences we tested for genetic differentiation among populations of $S$. cataractae. Thus, we performed an analysis of molecular variance (AMOVA) with the function poppr.amova in the poppr $\mathrm{R}$ package ${ }^{90}$, based on SNPs with no missing values ( $\mathrm{n}=23,252 \mathrm{SNPs}$ ). This function takes a genind object (created with the function df2genind from adegenet ${ }^{91}$; ploidy $=1$ ) as dependent variable and population (with 4 levels, $n=7-8$ samples per level) as predictor. The significance of the model was assessed using a randomization test with 9999 permutations on the output of the AMOVA (function randtest from the ade4 package ${ }^{92}$ ). Additionally, we calculated the likelihood ratio G-statistic to test for significant genetic differentiation among populations (gstats.glob function from the hierfstat package ${ }^{93}$ with 9999 permutations). We assessed its significance by comparing the observed statistic with the distribution of the G-statistic on a null dataset where samples were randomly shuffled among populations using the functionsamp.between from hierfstat (9999 repetitions).

Genetic data were visualized by means of principal component analysis (PCA) carried out with the function dudi.pca (ade4 package) on a scaled and centered allele frequency matrix obtained with the functionscaleGen (adegenet package).

\subsubsection{Epigenetic analyses}

We calculated the methylation level at each SMP and individual sample and estimated the mean and standard deviation of DNA methylation per group, for each separate sequence context (i.e. CG, CHG and CHH), and across all contexts, using the complete SMP matrix ( $\mathrm{n}=43,365$ SMPs) as well as the complete and polymorphic SMP matrix ( $\mathrm{n}=3,769$ SMPs). All other analyses were performed for each of the two common garden experiments by comparing control vs. Cd-treated and control vs. Cu-treated plants from all four populations using the matrix of complete and polymorphic SMPs.

To evaluate response to each heavy metal, we carried out a distance-based RDA (dbRDA ${ }^{94}$ ) to test the effect of population $(\mathrm{n}=4)$, treatment $(\mathrm{n}=2$; $\mathrm{C}$ vs. $\mathrm{Cd}$ or $\mathrm{C}$ vs. $\mathrm{Cu})$, and their interaction on genome wide DNA methylation with the model: epigenetic distance ${ }^{\sim}$ Population ${ }^{*}$ Treatment (functioncapscale in the vegan package; Oksanen et al., 2020). This constrained multivariate ordination approach provides an estimate of the amount of variation in epigenetic distances explained by our predictors, as well as the constrained ordination axes (RDA axes) used to visualize the structure of the data in a two-dimensional space. Pairwise epigenetic distances were estimated following Wang et al. ${ }^{96}$, where the distance $\left(\mathrm{d}_{\mathrm{st}}\right)$ between any two samples, $\mathrm{s}$ and $t$, was calculated either based on only the level of methylation or including the variance in methylation as follows:

$$
d_{\mathrm{st}}=\sqrt[2]{\sum_{j=1}^{n}\left\{\frac{1}{2 n}\left(x_{\mathrm{sj}}^{m}-x_{\mathrm{tj}}^{m}\right)^{2}+\frac{1}{2 n}\left(x_{\mathrm{sj}}^{v}-x_{\mathrm{tj}}^{v}\right)^{2}\right\}}
$$

Where $\mathrm{n}$ is the number of cytosine positions (SMPs); $x_{\mathrm{sj}}^{m}$ and $x_{\mathrm{tj}}^{m}$ represent the methylation level of cytosine $\mathrm{j}$ in samples $\mathrm{s}$ and $\mathrm{t}$ respectively; and $x_{\mathrm{sj}}^{v}$ and $x_{\mathrm{tj}}^{v}$ represent the methylation variance of cytosine $\mathrm{j}$ in samples $\mathrm{s}$ and $\mathrm{t}$ respectively. The methylation variance $\left(x_{\mathrm{ij}}^{v}\right)$ was estimated as the squared difference between the methylation level of cytosine $\mathrm{j}$ in sample $\mathrm{i}\left(x_{\mathrm{ij}}^{m}\right)$ and the average methylation of cytosine $\mathrm{j}$ across all samples in the group to which sample i belongs $\left(x_{j}^{m}\right)$. Using the formula above, we also accounted for the variation in methylation profiles within treatment groups.

We ran separate dbRDA models for each common garden experiment (i.e. $\mathrm{C}$ vs. $\mathrm{Cd}$ and $\mathrm{C}$ vs. $\mathrm{Cu}$ ) for all sequence contexts together and separately for each sequence context. In each of these cases, we first ran the model using the epigenetic distance matrix calculated using only differences in methylation levels between each pair of samples (using only the left-hand side under the square root of the formula above). Then, we ran the model using the distance matrix calculated from both methylation level and methylation variance (full formula). We tested the overall significance, as well as the significance of each predictor and 
their interaction using a permutation test with 9999 permutations. We calculated the proportion of total variance explained by our models, adjusted by the number of predictors included in the model (adj. $\mathrm{R}^{2}$ ), with the function RsquareAdj (vegan package) which provides a unique adj. $\mathrm{R}^{2}$ for the full model. We also calculated individual adj. $\mathrm{R}^{2}$ for each predictor by modelling the effect of each predictor while accounting for the others using partial constrained dbRDA with the following models: i) epigenetic distance Treatment + Condition(Population); ii) epigenetic distance $\sim$ Population + Condition(Treatment); iii) epigenetic distance $\sim$ Population:Treatment + Condition(Treatment) + Condition(Population).

We identified differentially methylated cytosine positions (DMPs) as cytosines with significant differences in their mean methylation level between control and $\mathrm{Cu}$-treated, and control and $\mathrm{Cd}$-treated plants using the R package DSS ${ }^{97}$. First, we modeled the methylation frequency at each cytosine position within each group using a beta-binomial distribution with arcsine link function and the formula " $0+$ group" (function DMLfit.multifactor ). This transformation proved useful to model DNA methylation because it reduces the heteroscedasticity of the data by stabilizing its variance ${ }^{98,99}$. Second, we performed Wald tests to detect differential methylation between groups at each position using the function DMLtest.multifactor which reports adjusted p-values by the Benjamini-Hochberg method (i.e. FDR). Cytosines were considered to be differentially methylated (i.e. DMPs) when the FDR [?] 0.05 and the methylation change was [?] $10 \%$.

\subsubsection{Gene expression analysis}

We identified differentially expressed transcripts (DETs) between control and Cu-treated plants from Sc3 and Sc4 using edgeR v3.24.3 ${ }^{100}$. First, we filtered low count transcripts by removing those with less than $2 \mathrm{cpm}$ (counts per million; i.e. less than 10 counts per transcript) in at least 3 samples to ensure that any transcript that was highly expressed in all three replicates from one group was kept. A total of 23,016 transcripts (7\% of the total) passed this filter and were used in subsequent steps. Second, we fitted the model using the function glmQLFit (option robust = TRUE) and tested for DETs using the function glm Treat . Transcripts were considered differentially expressed when the FDR $<0.001$ and the expression change was [?] 4 -fold $\left(\log _{2} \mathrm{FC}\right.$ [?] 2).

To better understand the changes associated with $\mathrm{Cu}$ exposure, we performed a Fisher's exact test (with the FatiGO package ${ }^{101}$ implemented within OmicsBox) on the list of DETs from each population. With this method, we tested for significant differences in the fraction of transcripts annotated with a specific GO term between the DETs list (test set) and the full list of annotated transcripts (reference set). We applied an FDR cutoff of 0.01 , and used the "Reduce to most specific" option within OmicsBox to remove the more general, less informative, GO terms.

\subsubsection{Association between DNA methylation and gene expression changes}

To find transcripts that were both differentially methylated and differentially expressed between $\mathrm{Cu}$-treated and control plants, i.e. potential associations between changes in DNA methylation and gene expression, we mapped the de novo reference to the de novotranscriptome using Blat ${ }^{102}$ and intersected IDs of the mapped sequences with the IDs of the lists of DMPs and DETs for each population.

\section{Results}

For the epiGBS libraries, we recovered 85.8 million reads after demultiplexing. The number of reads per sample averaged 2.5 million and ranged between 0.6-4.5 million. Sequenced reads were evenly distributed across all four populations ( $21.4 \pm 1.7$ million reads per population), and across all 12 groups $(7.2 \pm 2.1$ million reads per group) (Table S1). For the RNAseq libraries, we recovered 101.8 million reads after quality trimming. The number of reads per sample averaged 8.5 million and ranged between 6.9-10.2 million (Table S2). As for the epiGBS dataset, the sequencing power was evenly distributed across populations (51.0 and 50.8 million reads for $\mathrm{Sc} 3$ and $\mathrm{Sc} 4$ respectively), and the four groups (25.5 \pm 1.2 million reads per group) (Table S2).

The overall read mapping rate to the de novo transcriptome assembly ranged between $83-86 \%$ across samples (Table S3). We found a total of 390 complete orthologues, out of which $73 \%$ were complete and single copy. 
In total, 26,638 transcripts were successfully annotated. A summary of the main GO terms in the annotated transcriptome is shown in Table S3.

\subsection{Genetic structure}

We found no evidence of genetic differentiation among populations of the copper moss $S$. cataractae . The AMOVA test showed a very low percent of genetic variation among populations $(0.01 \% ; \varphi=0.0001)$, and the randomization test performed on the output of the AMOVA was not significant ( $\mathrm{p}$-value $=0.462$ ). Similarly, the permutation test carried out to assess the significance of the G-statistic was not significant (G-stat = $83712.2 ; \mathrm{p}=0.839)$. The lack of genetic differences among populations is shown in Fig. S1.

\subsection{Epigenetic changes in response to $\mathrm{Cd}$ and $\mathrm{Cu}$}

Overall, DNA methylation in S. cataractae was very low (Table 1). For the complete SMP matrix (n=43,365 SMPs) mean cytosine methylation per group averaged $3.3 \% \pm 0.06$ in all contexts considered together, and $0.69 \% \pm 0.05$ in CG, $12 \% \pm 0.05$ in CHG, and $0.71 \% \pm 0.07$ in CHH. Most of the cytosines in the CG, CHG, and $\mathrm{CHH}$ contexts were unmethylated, i.e. methylation level [?] $5 \%$ in [?] $95 \%$ of the samples (92.6\% in CG, 91.2\% in $\mathrm{CHH}$ and $81.9 \%$ in $\mathrm{CHG}$ ); $1.9 \%$ of the cytosines in the CHG context were fully methylated, i.e. methylation level [?] $95 \%$ in [?] $95 \%$ of the samples (Fig. S2A). The standard deviation of DNA methylation averaged $16.0 \%$ +- 0.07 in all contexts together, and $3.1 \%+-0.39$ in CG, $31.5 \%+-0.05$ in CHG, and $2.8 \%$ +- 0.39 in CHH (Table 1). For the complete and polymorphic SMP matrix ( $\mathrm{n}=3,769$ SMPs), mean cytosine methylation per group averaged $10.8 \%+-0.25$ in all contexts, and $2.8 \%+-0.26$ in CG, $34.3 \%+-0.23$ in CHG, and 2.4\% +- 0.32 in CHH (Fig. S2B). The standard deviation of DNA methylation averaged $27.5 \%$ +- 0.15 in all contexts, and $7.2 \%$ +- 0.88 in CG, $45.3 \%$ +- 0.18 in CHG, and $5.3 \%$ +- 0.86 in CHH (Table 1).

DNA methylation levels differed among genomic features with repeats generally showing higher methylation levels than genes and transposons; for example, mean methylation across samples in repeats was 0.9, 12.6, and $1 \%$ in the $\mathrm{CG}, \mathrm{CHG}$, and $\mathrm{CHH}$ contexts respectively compared to the $0.5,10.2$, and $0.6 \%$ in the CG, $\mathrm{CHG}$, and CHH contexts found for genes and transposons respectively (Fig. S3).

The dbRDA performed to test the effect of population, treatment, and their interaction on genome wide DNA methylation in $S$. cataractaewas not significant when run with the epigenetic distance matrix calculated comparing the methylation level only (Table S4). When run with the distance matrix obtained comparing both methylation level and methylation variance between samples, the model was significant for both common garden experiments, and explained $61 \%$ and $56 \%$ of the variation of the epigenetic distances in the Cd vs. C and the $\mathrm{Cu}$ vs. $\mathrm{C}$ comparison respectively (Table 2 ; results for each separate sequence context were similar and are shown in Table S4). The effect of both predictors and their interaction was significant in both experiments: treatment explained $\sim 9 \%$ and $7 \%$ of the variation of the epigenetic distances in the $\mathrm{Cd}$ vs. C and $\mathrm{Cu}$ vs. $\mathrm{C}$ comparisons respectively; population explained $\sim 21 \%$ of the variation in both experiments; and the interaction explained $33 \%$ and $29 \%$ of the variation in the $\mathrm{Cd}$ vs. $\mathrm{C}$ and $\mathrm{Cu}$ vs. C comparisons respectively (Table 2). Both metal treatments induced more DNA methylation variability among samples of Sc2 (one of the most metal tolerant populations); this effect was weaker, yet noticeable on Sc1 and Sc2 in response to $\mathrm{Cu}$, and on Sc4 in response to $\mathrm{Cd}$ (Fig. 1A1, A2). The first two constrained axes of the dbRDA explained $\sim 35 \%$ and $\sim 29 \%$ of the variation in epigenetic distances in the $\mathrm{Cd}$ vs. $\mathrm{C}$ and the $\mathrm{Cu}$ vs. $\mathrm{C}$ comparison respectively. Most of the variation in the first axis (RDA1) was driven by the differentiation of treated samples from Sc2 in both common garden experiments, whereas most of the variation in the second axis (RDA2) was driven by the differentiation of control samples from Sc3 and Sc4 in the two common garden experiments (Fig.1A1, A2).

We found between 25-78 differentially methylated positions (DMPs) between Cd-treated and control (Fig. 1B1) and $\mathrm{Cu}$-treated and control plants (Fig. 1B2) across populations (between 0.66-2.1\% of the polymorphic cytosines; Fig. S4). All populations showed more DMPs within the CHH context $(17 \%, 26 \%$, and $57 \%$ of all DMPs in CG, CHG, and CHH contexts respectively; Table S5) but the proportion of DMPs per context did not differ from the proportion of cytosines interrogated per context. Sc3 showed the highest number of 
DMPs in both treatments (Figs. 1B1, 1B2, S4). Cadmium induced more DMPs than Cu (except in Sc4; Figs. 1B1, 1B2, S4). We found more DMPs with a negative methylation change, i.e. hypomethylation, in $\mathrm{Sc} 2$ in response to $\mathrm{Cd}$ and $\mathrm{Cu}$, and in $\mathrm{Sc} 3$ in response to $\mathrm{Cu}$; $\mathrm{Sc} 1$ and $\mathrm{Sc} 4$ showed more DMPs with a positive methylation change, i.e. hypermethylation, in response to $\mathrm{Cd}$ and $\mathrm{Cu}$ respectively. Most DMPs were unique for each population except four: two shared between Sc1 and Sc2 and one shared between Sc1 and Sc4 in the $\mathrm{Cd}$ vs. C comparison; one shared between Sc3 and Sc4 in the $\mathrm{Cu}$ vs. C comparison (Fig. S5A, S5B). The two metals, however, induced a considerable number of common DMPs within each population, most of which were hypomethylated: $29,49,41$, and $12 \%$ of the DMPs were common to both treatments in Sc1 to Sc4 respectively (Fig. S5C, underlined DMPs in Table S5).

\subsection{Effect of $C u$ on gene expression}

The transcript expression profiles of the plants were significantly affected by $\mathrm{Cu}$ exposure in the two studied populations. The effect of $\mathrm{Cu}$ was greater in the most tolerant population (Sc3) as shown by: (i) the greatest separation between control and Cu-treated samples of Sc3 in the multidimensional scaling plot (Fig. 1C1); (ii) the greater number of DETs in Sc3 than in Sc4, i.e. 1,710 DETs in Sc3 - 7.4\% of the total - and 259 DTEs in Sc4 - 1.1\% of the total; and (iii) the greater expression changes shown by DETs in Sc3 compared to the same DETs in Sc4 (Fig. 1C2; Fig. S6). The majority of the DETs found in each population were down-regulated, i.e. $99 \%$ and $95 \%$ in Sc3 and Sc4 respectively (Table S6), indicating that these transcripts were constitutively expressed in control plants, and their expression was significantly repressed in response to $\mathrm{Cu}$. The direction of the expression change, i.e. up- or downregulation, of the 220 DETs that were common to both populations was the same; the intensity of the change, however, was [?] 2 times higher in Sc3 for $62 \%$ of the common DETs (on average, FC in this fraction of DETs was 9x higher in Sc3 compared to Sc4); the opposite was true in only $9 \%$ of the common DETs (with an average FC in this fraction of DETs 3.4x higher in Sc4 than Sc3) (Fig. 1C2, S6, Table S6).

In total, $74 \%$ and $76 \%$ of the DETs were annotated in Sc3 and Sc4 respectively (Table S6). The results of the Fisher's exact test showed 117 over-represented and 13 under-represented GO terms in Sc3, and 11 overrepresented GO terms in Sc4 in the lists of DETs compared to the full list of transcripts (Table S7). The most over-represented molecular functions in Sc3 included terms related to RNA metabolism and ribosomal structure (RNA binding, GO:0003723; structural constituent of ribosome, GO:0003735), protein molecular interactions (protein-containing complex binding GO:0044877; protein homodimerization activity; GO:0042803; protein N-terminus binding, GO:0047485), glycosidic bond hydrolysis (hydrolase activity, hydrolyzing Oglycosyl compounds, GO:0004553), protein hydrolysis and its regulation (exopeptidase activity, GO:0008238; serine-type endopeptidase activity, GO:0004252; endopeptidase inhibitor activity, GO:0004866), redox activity (oxidoreductase activity, GO:0016715), and Ca signaling/regulation (Ca ion binding, GO:0005509) (Fig. 2A). These functions were only represented within the down-regulated DETs list (except Ca ion binding, also present within the up-regulated DETs; Table S7). Over-represented molecular functions in Sc4 consisted of protein hydrolysis (cysteine-type endopeptidase activity, GO:0004197; serine-type endopeptidase activity, GO:0004252), chitin binding (GO:0008061), and structural molecule activity (GO:0005198) (Fig. 2B), all of which were only represented in the down-regulated DETs list (Table S5). The GO terms related to biological process, molecular function, and cellular component associated to all DETs of each population are shown in Fig. S7.

We classified down-regulated DETs from Sc3 and Sc4 into 13 different functional groups according to their annotation descriptions (Table S8; Fig. 2C,D) which included transcripts related to: i) protein and ribosome biosynthesis, including structural constituents of ribosomal subunits as well as transcripts involved in protein transcription, translation and elongation; ii) protein degradation and/or turnover, including many proteases, transcripts involved in protein ubiquitination, and structural components and regulators of the proteasome; iii) transcripts with reduction-oxidation (redox) activity, including components of the reactive oxygen species (ROS) scavenging systems (e.g. glutathione S-transferase, ascorbate peroxidase, catalase); iv) protein repair and proper protein folding activity, mostly comprised by protein chaperones; v) Ca homeostasis and signal transduction including Ca-transporting ATPases, Ca calmodulin-dependent kinases, and 
calmodulin; vi) energy metabolism including enzymes involved in glycolysis, pentose-phosphate, and tricarboxylic acid cycle pathways; vii) transmembrane transport like V-type proton ATPases, ABC transporters, or zinc finger proteins; viii) RNA metabolism, including RNA helicases, mRNA splicing factors, and rRNA processing factors. The remaining groups included transcripts related to vesicle trafficking, fatty acid oxidation, chromatin organization, polyamine biosynthesis and degradation, and purine metabolism. For Sc4, all transcripts except those involved in vesicle trafficking, fatty acid oxidation, and chromatin organization, were represented within the down-regulated DETs list, although the number of transcripts within each group was significantly lower (Fig. 2D; Table S8).

Up-regulated transcripts in Sc3 included enzymes involved in the regulation of the redox state of the cells (probable 2-oxoglutarate-dependent dioxygenase ANS, L-ascorbate oxidase-like, cytokinin hydroxylase, and peroxidase P7-like), and transcripts were involved in cellular signaling processes (EG45-like domain containing protein) and lipid hydrolysis (GDSL esterase/lipase). Three transcripts were up-regulated both in Sc3 and Sc4: allene oxide cyclase, an enzyme catalyzing the most important step in the jasmonic acid (JA) biosynthetic pathway; CML25, a member of the calmodulin-like protein group; and L-ascorbate oxidaselike protein, involved in the regulation of the redox status of the cells. Uniquely up-regulated DETs in Sc4 included the metal homeostasis factor ATX1-like, a copper chaperone that delivers $\mathrm{Cu}$ to heavy metal P-type ATPases, two probable copper-transporting ATPases (HMA5), involved in copper transport across membranes, two dehydrogenases with oxidoreductase activity (alcohol dehydrogenase 1-like; and aldehyde dehydrogenase family 2 member B7, mitochondrial-like), and one primary amine oxidase-like, involved in polyamine degradation (descriptions as well as expression changes of all DETs are present in Table S6).

Finally, we found 100 DETs between control plants from Sc3 and Sc4; 68 DETs were significantly upregulated in Sc3 whereas 32 were downregulated in these plants compared to those from Sc4. Annotated upregulated DETs showed a greater expression of components of the protein biosynthesis and degradation machinery and signal transduction, which were downregulated in response to $\mathrm{Cu}$ in both populations. Annotated downregulated DETs, on the other hand, were related to the energy metabolism and vacuole transport, among others (Table S6)

\subsection{Relationship between DNA methylation and expression changes}

A total of 12,631 contigs from the de novo genome mapped to 8,623 transcripts but none of the DMPs mapped to any of the DETs. Yet, multiple contigs with DMPs mapped to transcripts that were annotated; descriptions of these sequences matched some of the main processes down-regulated during Cu-exposure such as protein degradation/turnover, chromatin organization, membrane transporters or signal transduction (Table S4).

\section{Discussion}

In this work, we used epiGBS and RNAseq to study the drivers of intraspecific variation for heavy metal tolerance in the copper moss $S$. cataractae, and to provide new insights into the molecular mechanisms used by bryophytes to deal with heavy metal toxicity. Our results showed that (i) genetic differentiation did not explain phenotypic differences in $\mathrm{Cd}$ and $\mathrm{Cu}$ tolerance in $S$. cataractae; (ii) heavy metal exposure induced some DNA methylation changes. These, however, were inconsistent within treatment groups leading to an increase in methylation variation in $\mathrm{Cd}$ - and/or $\mathrm{Cu}$-treated plants in plants from the most tolerant populations; (iii) single cytosine methylation changes (SMPs) tended to hypomethylation in the most tolerant populations and to hypermethylation in the least tolerant ones; (iv) plants from both the more and the less tolerant populations constitutively expressed multiple genes involved in heavy metal tolerance in the absence of $\mathrm{Cu}$ in the laboratory. The most tolerant plants, however, expressed more genes involved in the alleviation of heavy metal stress under control conditions, whereas the less tolerant invested more in growth; and v) upon acute $\mathrm{Cu}$ stress, both populations inhibited the expression of these protective genes to nearly equivalent levels. The magnitude of this inhibition was greater in the more tolerant population. Altogether, these results suggest that chronic exposure to different levels of heavy metals in the field is associated with non-geneticallybased intraspecific differentiation for heavy metal tolerance in $S$. cataractae. At the molecular level, this 
phenotypic differentiation was reflected in a greater constitutive investment in protective mechanisms (e.g. ROS scavenging enzymes, heavy metal transporters, protein chaperones) in the most tolerant population, but maintaining an equivalent response into an energy conservative state when facing an acute $\mathrm{Cu}$ stress event in the laboratory.

Scopelophila cataractae is called "copper moss" due to its high affinity for heavy metals, especially Cu. In fact, its worldwide geographical distribution mostly matches that of $\mathrm{Cu}$-enriched substrates ${ }^{72,103,104}$, and its growth has been consistently favored in the presence of $\mathrm{Cu}$ in the laboratory ${ }^{105,106}$. Yet, previous studies have shown that this metal compromises the species' growth upon exposure to high concentrations of bioavailable $\mathrm{Cu}{ }^{68,105}$, and that this acute $\mathrm{Cu}$ stress can uncover novel intraspecific variation in $\mathrm{Cu}$ tolerance ${ }^{68}$. In our previous study, we reported phenotypic differentiation for $\mathrm{Cd}$ and $\mathrm{Cu}$ tolerance among plants from the same populations studied here: growth of plants from populations that originated from contaminated microhabitats was increased (Sc2) or unaffected (Sc3) in metal-treated compared to control plants, whereas growth of plants from populations that originated from contaminated microhabitats (Sc3, Sc4) was reduced 68. These differences were observed after clonally propagating all plants in control conditions for months, which should have eliminated any potential carryover environmental effects from the field. Taken together, these findings supported a role of natural selection in generating locally adapted genotypes among the four populations. Nonetheless, our current results showed no evidence of genetic differentiation among the populations studied, at least in the portion of the genome interrogated with the epiGBS technique. The lack of genetic differentiation could be explained by the movement of gametophore fragments and asexual propagules down the slope of the field site ( $500 \mathrm{~m}$ long). Thus, our analysis suggested that this phenotypic differentiation could have arisen through mechanisms other than DNA sequence variation.

Epigenetic variation has emerged in the past decades as a potentially important source of phenotypic variation and differentiation in plants ${ }^{107-113}$. Thus, we assessed whether DNA methylation contributed to the differences in tolerance observed in our common garden experiments. We did not find evidence for epigenetic differentiation among populations when comparing mean methylation levels across all cytosines, and the effect of $\mathrm{Cd}$ and $\mathrm{Cu}$ exposure on individual cytosine methylation levels was rather limited compared to response to other stresses in other plants (e.g. ${ }^{114}$ ). We propose three possible causes for this limited response. First of all, environmental stress can increase inter-individual methylation variation in plants ${ }^{115}$. This type of inconsistency would limit our capacity to find clear directional changes between experimental groups. In this study, we found a significant increase in DNA methylation variability in plants from Sc2 in response to both metal treatments, and a slight increase in variability in plants from $\mathrm{Sc} 1$ and $\mathrm{Sc} 3$ in response to $\mathrm{Cu}$, and from Sc4 in response to $\mathrm{Cd}$. Although this effect needs to be further validated with a greater number of replicate samples per treatment group, these results support a potential population-specific epigenetic response to heavy metal exposure. Even though it has not been experimentally proven, such stress-induced inter-individual epigenetic variation could contribute to increased population-level phenotypic variation and help plant populations to deal with environmental stress ${ }^{116}$.

On the other hand, the limited epigenetic response found here could also be due, in part, to fundamental differences between the epigenomic landscape of bryophytes and angiosperms, combined with the technical characteristics of the epiGBS technique. Overall, bryophyte genomes are much less methylated than angiosperm genomes (117,118, but see ${ }^{119}$ for differences among stages of the bryophyte life cycle), and cytosine methylation tends to be segregated away from genic regions ${ }^{120}$. For example, in contrast to flowering plants, gene body methylation (gbM) is absent in most genes of the model moss $P$. patens ${ }^{117}$ and the model liverwort Marchantia polymorpha ${ }^{118}$ (but see ${ }^{119}$ ). The epiGBS technique interrogates a biased subset of the genome, as the restriction enzyme that we used, PstI, preferentially targets coding regions ${ }^{71}$. Together with the low gbM found for other bryophytes, this would explain the very low DNA methylation levels found here, and the limited response to heavy metal exposure within the interrogated genomic regions. Further, our epiGBS fragments did not overlap with our DETs so we had no information about the methylation status of those specific transcripts (as reported in ${ }^{121,122}$. Finally, it is also possible that epigenetic mechanisms other than DNA methylation, or even point mutations located in genomic regions other than that interrogated here, could determine the phenotypic differences observed here. For example, the population-specific expression 
of proteins involved in chromatin reorganization (only differentially expressed in the more tolerant population), like the histone modifying enzyme histone deacetylase 6 and its associated WD-40 repeat-containing protein MSI1, the histone chaperone peptidyl-prolyl cis-trans isomerase FKBP53, and the SWI/SNF-related matrix-associated actin-dependent regulator of chromatin subfamily A member 5, points towards a potential role of histone modifications in heavy metal stress tolerance in $S$. cataractae .

Some authors pointed out that changes in DNA methylation in response to heavy metal stress could be due to a side effect of metal-induced ROS production ${ }^{42}$. However, some of the patterns observed here indicate otherwise. First, exposure to $\mathrm{Cd}$ induced more methylation changes despite the lack of a significant increase in oxidative damage in any of the populations studied (Fig. $3 \mathrm{H}$ in ${ }^{68}$ ). Second, the effect of $\mathrm{Cd}$ and $\mathrm{Cu}$ on DNA methylation was population-specific, with more methylation variability induced in one of the most tolerant moss populations (Sc2). Third, more cytosines were hypo- than hypermethylated in plants from the most tolerant populations (Sc2, Sc3), while we observed the opposite trend in the less tolerant ones (Sc1, Sc4). Finally, when compared within each population, both metals induced numerous common DMPs. Altogether, these findings suggest that the DNA methylation changes observed here were not random, as expected if generated through metal-induced ROS production, but likely dependent on the previous stress experience of the plants.

The set of DETs found in this study provided us with valuable information about the adaptive mechanisms to deal with chronic $\mathrm{Cu}$ exposure developed by $S$. cataractae in natural conditions. For example, $16.2 \%$ and $23.6 \%$ of all DETs in Sc3 and Sc4 respectively, were involved in cellular protein homeostasis including protein and RNA biosynthesis/degradation, and protein repair. Heavy metals can disrupt protein folding and cause aggregation 10,123-125. Aggregation and accumulation of unfolded proteins triggers the unfolded protein response (UPR) pathway, which enhances protein folding and degradation, and reduces protein production under stress conditions ${ }^{123}$. Multiple components of the UPR pathway were constitutively expressed in $S$. cataractae, including components of the calnexin/calreticulin complex as well as protein chaperones involved in protein folding, the Ubiquitin-26S proteasome proteolytic system, and multiple translation and elongation initiation factors involved in protein synthesis.

Similarly, a considerable number of constitutively expressed transcripts were involved in direct or indirect ROS detoxification (e.g. glutathione S-transferases, L-ascorbate peroxidases, superoxide dismutases), cellular signaling, trafficking and metal transport (e.g. calmodulins, clathrins, ras-related proteins, V-type proton ATPases, zinc finger proteins, ABC transporters), fatty acid oxidation enzymes related to the jasmonic acid biosynthetic pathway (e.g. peroxisomal acyl-CoA oxidase, 3-ketoacyl-CoA thiolase), and purine metabolism (adenosine kinases, inosine-5'-monophosphate dehydrogenase). Polyamine metabolism, including enzymes like ornithine decarboxylase, S-adenosylmethionine synthase, and spermidine synthase, was also associated with the adaptive response to $\mathrm{Cu}$ in $S$. cataractae. These organic compounds have been shown to increase heavy metal tolerance in plants through ROS detoxification ${ }^{126-128}$ and decreased metal accumulation ${ }^{126,129}$. The functioning of all these stress alleviation mechanisms is energy and carbon consuming, which explains the increased constitutive expression of enzymes involved in energy production like the glycolysis, penthosephosphate, and tricarboxylic acid pathways ${ }^{130}$.

In this study, we found evidence of potentially non-genetically-based phenotypic differentiation for heavy metal tolerance in bryophytes. Our results indicated that chronic exposure to high concentrations of metals in natural conditions led to constitutive overexpression of genes that contribute to heavy metal stress tolerance in several ways (e.g. regulation of cellular protein homeostasis, ROS detoxification, metal transport). Importantly, plants that had grown under higher stress conditions in the field were more tolerant to acute $\mathrm{Cu}$ stress events like that used in our experiments, possibly due to their greater capacity to decrease production of energetically expensive products as a strategy to reduce energy consumption in a situation in which the ability of these mechanisms to provide protection against the stress would be overwhelmed. Based on the higher epigenetic response of the most tolerant populations (greater changes in DNA methylation and constitutive expression of chromatin remodeling proteins) we propose that their increased capacity to regulate the expression of heavy metal stress-related genes could have an epigenetic basis that we could not detect 
with our approach. This work thus serves as a proof of concept for future studies which should focus on the targeted evaluation of the regulatory mechanisms presented here, and evaluate their relative importance in heavy metal stress tolerance in bryophytes.

\section{Author contributions}

MTB, CLR, and CA conceived and designed the experiments; MTB, SC, and NCAM performed the wet lab work; MTB and MWS performed the data analyses; MTB wrote first draft of the manuscript; MTB, CA, CLR, SFM, SC, MWS, and NCAMW critically reviewed and edited the manuscript.

\section{Conflicts of interest}

The authors declare that there are no conflicts of interest.

\section{Data availability}

The pipeline scripts used for this study are available at: https://github.com/thomasvangurp/epiGBS, with a bug-fix modification (https://github.com/MWSchmid/epiGBS_Nov_2017_fixed). The raw sequence data files for epiGBS (Illumina paired end reads), and for RNAseq (individual samples' Illumina paired end reads after quality trimming) have been submitted to the Sequence Read Archive (SRA) of NCBI under project number PRJNA790924. The barcodes (barcodes.tsv) required to process the raw epiGBS sequencing data available on SRA, the reference contigs (de_novo_contigs.fasta.gz), the annotation of the reference contigs (mergedAnnot.csv.gz), the SNPs (snps.vcf.gz), the methylation data (methylation.txt.gz), and the de novo transcriptome assembly (de_novo_transcriptome.fasta.gz) are available on zenodo (doi: 10.5281/zenodo.5776915).

\section{References}

1. Ali, H., Khan, E. \& Ilahi, I. Environmental Chemistry and Ecotoxicology of Hazardous Heavy Metals: Environmental Persistence, Toxicity, and Bioaccumulation. Journal of Chemistry2019, e6730305 (2019).

2. He, Z. L., Yang, X. E. \& Stoffella, P. J. Trace elements in agroecosystems and impacts on the environment. J Trace Elem Med Biol 19 , 125-140 (2005).

3. Kanawade, S. M., Hamigi, A. D. \& Gaikwad, R. W. Ecological Effect of Pollution. IJCEA 332-335 (2010) doi:10.7763/IJCEA.2010.V1.57.

4. Rhind, S. M. Anthropogenic pollutants: a threat to ecosystem sustainability? Philos Trans R Soc Lond B Biol Sci 364 , 3391-3401 (2009).

5. Brown, D. H. \& Wells, J. M. Physiological Effects of Heavy Metals on the Moss Rhytidiadelphus squarrosus. Annals of Botany66 , 641-647 (1990).

6. Devi, S. R. \& Prasad, M. N. V. Membrane Lipid Alterations in Heavy Metal Exposed Plants. in Heavy Metal Stress in Plants: From Molecules to Ecosystems (eds. Prasad, M. N. V. \& Hagemeyer, J.) 99-116 (Springer, 1999). doi:10.1007/978-3-662-07745-0_5.

7. Guschina, I. A. \& Harwood, J. L. Lipid metabolism in the moss Rhytidiadelphus squarrosus (Hedw.) Warnst. from lead-contaminated and non-contaminated populations. Journal of Experimental Botany53 , 455-463 (2002).

8. Janicka-Russak, M., Kabała, K., Burzyński, M. \& Kłobus, G. Response of plasma membrane H+-ATPase to heavy metal stress in Cucumis sativus roots. Journal of Experimental Botany 59, 3721-3728 (2008).

9. Boominathan, R. \& Doran, P. M. Ni-induced oxidative stress in roots of the Ni hyperaccumulator, Alyssum bertolonii. New Phytologist156 , 205-215 (2002).

10. Shahid, M. et al. Heavy-Metal-Induced Reactive Oxygen Species: Phytotoxicity and Physicochemical Changes in Plants. inReviews of Environmental Contamination and Toxicology Volume 232(ed. Whitacre, D. M.) 1-44 (Springer International Publishing, 2014). doi:10.1007/978-3-319-06746-9_1. 
11. Assche, F. V. \& Clijsters, H. Effects of metals on enzyme activity in plants. Plant, Cell \& Environment 13, 195-206 (1990).

12. Küpper, H., Dědic, R., Svoboda, A., Hála, J. \& Kroneck, P. M. H. Kinetics and efficiency of excitation energy transfer from chlorophylls, their heavy metal-substituted derivatives, and pheophytins to singlet oxygen. Biochimica et Biophysica Acta (BBA) - General Subjects1572 , 107-113 (2002).

13. Küpper, H., Šetlík, I., Spiller, M., Küpper, F. C. \& Prášil, O. Heavy Metal-Induced Inhibition of Photosynthesis: Targets of in Vivo Heavy Metal Chlorophyll Formation1. Journal of Phycology38 , 429-441 (2002).

14. Tan, Y.-F., O’Toole, N., Taylor, N. L. \& Millar, A. H. Divalent Metal Ions in Plant Mitochondria and Their Role in Interactions with Proteins and Oxidative Stress-Induced Damage to Respiratory Function.Plant Physiol 152, 747-761 (2010).

15. Verbruggen, N., Hermans, C. \& Schat, H. Molecular mechanisms of metal hyperaccumulation in plants. New Phytologist 181, 759-776 (2009).

16. Krämer, U. Metal Hyperaccumulation in Plants. Annu. Rev. Plant Biol. 61, 517-534 (2010).

17. Papadopulos, A. S. T. et al. Rapid Parallel Adaptation to Anthropogenic Heavy Metal Pollution. Molecular Biology and Evolution $38,3724-3736$ (2021).

18. Becher, M., Talke, I. N., Krall, L. \& Krämer, U. Cross-species microarray transcript profiling reveals high constitutive expression of metal homeostasis genes in shoots of the zinc hyperaccumulator Arabidopsis halleri. The Plant Journal 37 , 251-268 (2004).

19. Gao, J., Sun, L., Yang, X. \& Liu, J.-X. Transcriptomic Analysis of Cadmium Stress Response in the Heavy Metal Hyperaccumulator Sedum alfredii Hance. PLoS One 8, e64643 (2013).

20. Halimaa, P. et al. Gene Expression Differences betweenNoccaea caerulescens Ecotypes Help to Identify Candidate Genes for Metal Phytoremediation. Environ. Sci. Technol. 48 , 3344-3353 (2014).

21. Meier, S. K. et al. Comparative RNA-seq analysis of nickel hyperaccumulating and non-accumulating populations of Senecio coronatus (Asteraceae). The Plant Journal 95 , 1023-1038 (2018).

22. Pence, N. S. et al. The molecular physiology of heavy metal transport in the $\mathrm{Zn} / \mathrm{Cd}$ hyperaccumulator Thlaspi caerulescens.PNAS 97 , 4956-4960 (2000).

23. van de Mortel, J. E. et al. Large Expression Differences in Genes for Iron and Zinc Homeostasis, Stress Response, and Lignin Biosynthesis Distinguish Roots of Arabidopsis thaliana and the Related Metal Hyperaccumulator Thlaspi caerulescens. Plant Physiol142, 1127-1147 (2006).

24. Hanikenne, M. et al. Evolution of metal hyperaccumulation required cis-regulatory changes and triplication of HMA4. Nature 453 , 391-395 (2008).

25. Talke, I. N., Hanikenne, M. \& Krämer, U. Zinc-Dependent Global Transcriptional Control, Transcriptional Deregulation, and Higher Gene Copy Number for Genes in Metal Homeostasis of the Hyperaccumulator Arabidopsis halleri. Plant Physiol 142, 148-167 (2006).

26. Bartee, L., Shriner, W. \& Creech, C. Eukaryotic epigenetic regulation. (2017).

27. Li, B., Carey, M. \& Workman, J. L. The Role of Chromatin during Transcription. Cell 128 , 707-719 (2007).

28. Gehring, M., Bubb, K. L. \& Henikoff, S. Extensive Demethylation of Repetitive Elements During Seed Development Underlies Gene Imprinting.Science 324, 1447-1451 (2009).

29. Lang, Z. et al. Critical roles of DNA demethylation in the activation of ripening-induced genes and inhibition of ripening-repressed genes in tomato fruit. Proc Natl Acad Sci U S A 114, E4511-E4519 (2017). 
30. Rathore, P., Raina, S. N., Kumar, S. \& Bhat, V. Retro-Element Gypsy-163 Is Differentially Methylated in Reproductive Tissues of Apomictic and Sexual Plants of Cenchrus ciliaris. Frontiers in Genetics 11, 795 (2020).

31. Sanchez, D. H. \& Paszkowski, J. Heat-Induced Release of Epigenetic Silencing Reveals the Concealed Role of an Imprinted Plant Gene.PLOS Genetics 10 , e1004806 (2014).

32. Tsuji, H., Saika, H., Tsutsumi, N., Hirai, A. \& Nakazono, M. Dynamic and Reversible Changes in Histone H3-Lys4 Methylation and H3 Acetylation Occurring at Submergence-inducible Genes in Rice.Plant and Cell Physiology 47, 995-1003 (2006).

33. Zhang, M., Xu, C., von Wettstein, D. \& Liu, B. Tissue-Specific Differences in Cytosine Methylation and Their Association with Differential Gene Expression in Sorghum1[W]. Plant Physiol156 , 1955-1966 (2011).

34. Galati, S. et al. Heavy metals modulate DNA compaction and methylation at CpG sites in the metal hyperaccumulator Arabidopsis halleri. Environmental and Molecular Mutagenesis 62 , 133-142 (2021).

35. Gullì, M., Marchi, L., Fragni, R., Buschini, A. \& Visioli, G. Epigenetic modifications preserve the hyperaccumulator Noccaea caerulescens from Ni geno-toxicity. Environmental and Molecular Mutagenesis 59, $464-475$ (2018).

36. Ghosh, I., Sadhu, A., Moriyasu, Y., Bandyopadhyay, M. \& Mukherjee, A. Manganese oxide nanoparticles induce genotoxicity and DNA hypomethylation in the moss Physcomitrella patens. Mutation Research/Genetic Toxicology and Environmental Mutagenesis 842 , 146-157 (2019).

37. Pour, A. H., Özkan, G., Nalci, Ö. B. \& HaliLoğlu, K. Estimation of genomic instability and DNA methylation due to aluminum (Al) stress in wheat (Triticum aestivum L.) using iPBS and CRED-iPBS analyses. Turk J Bot 43, 27-37 (2019).

38. Taspinar, M. S. et al. Aluminum-Induced Changes on DNA Damage, DNA Methylation and LTR Retrotransposon Polymorphism in Maize.Arab J Sci Eng 43, 123-131 (2018).

39. Agar, G. et al. Effects of Lead Sulfate on Genetic and Epigenetic Changes, and Endogenous Hormone Levels in Corn ( Zea mays L.). Pol. J. Environ. Stud. 23 , 1925-1932 (2014).

40. Greco, M., Chiappetta, A., Bruno, L. \& Bitonti, M. B. In Posidonia oceanica cadmium induces changes in DNA methylation and chromatin patterning. J Exp Bot 63 , 695-709 (2012).

41. Xin, C., Chi, J., Zhao, Y., He, Y. \& Guo, J. Cadmium stress alters cytosine methylation status and expression of a select set of genes in Nicotiana benthamiana. Plant Science 284 , 16-24 (2019).

42. Aina, R. et al. Specific hypomethylation of DNA is induced by heavy metals in white clover and industrial hemp. Physiologia Plantarum 121, 472-480 (2004).

43. Filek, M. et al. The protective role of selenium in rape seedlings subjected to cadmium stress. Journal of Plant Physiology 165, 833-844 (2008).

44. Kumar, M., Bijo, A. J., Baghel, R. S., Reddy, C. R. K. \& Jha, B. Selenium and spermine alleviate cadmium induced toxicity in the red seaweed Gracilaria dura by regulating antioxidants and DNA methylation.Plant Physiology and Biochemistry 51, 129-138 (2012).

45. Bednarek, P. T., Orłowska, R. \& Niedziela, A. A relative quantitative Methylation-Sensitive Amplified Polymorphism (MSAP) method for the analysis of abiotic stress. BMC Plant Biology 17 , 79 (2017).

46. Niedziela, A. The influence of Al3+ on DNA methylation and sequence changes in the triticale $(\times$ Triticosecale Wittmack) genome. J Appl Genetics 59 , 405-417 (2018).

47. Cicatelli, A. et al. Epigenetic control of heavy metal stress response in mycorrhizal versus non-mycorrhizal poplar plants.Environ Sci Pollut Res 21 , 1723-1737 (2014). 
48. Feng, S. J., Liu, X. S., Cao, H. W. \& Yang, Z. M. Identification of a rice metallochaperone for cadmium tolerance by an epigenetic mechanism and potential use for clean up in wetland. Environmental Pollution 288, 117837 (2021).

49. Feng, S. J. et al. Identification of epigenetic mechanisms in paddy crop associated with lowering environmentally related cadmium risks to food safety. Environmental Pollution 256 , 113464 (2020).

50. Ezaki, B., Higashi, A., Nanba, N. \& Nishiuchi, T. An S-adenosyl Methionine Synthetase (SAMS) Gene from Andropogon virginicus L. Confers Aluminum Stress Tolerance and Facilitates Epigenetic Gene Regulation in Arabidopsis thaliana. Frontiers in Plant Science 7 , 1627 (2016).

51. Shafiq, S. et al. Lead, Cadmium and Zinc Phytotoxicity Alter DNA Methylation Levels to Confer Heavy Metal Tolerance in Wheat.Int J Mol Sci 20 , (2019).

52. Dubey, S. et al. Identification and expression analysis of conserved microRNAs during short and prolonged chromium stress in rice (Oryza sativa). Environ Sci Pollut Res 27 , 380-390 (2020).

53. Ding, Y., Chen, Z. \& Zhu, C. Microarray-based analysis of cadmium-responsive microRNAs in rice (Oryza sativa). J Exp Bot62 , 3563-3573 (2011).

54. Huang, S. Q., Peng, J., Qiu, C. X. \& Yang, Z. M. Heavy metal-regulated new microRNAs from rice. Journal of Inorganic Biochemistry 103, 282-287 (2009).

55. Huang, S. Q. et al. A set of miRNAs from Brassica napus in response to sulphate deficiency and cadmium stress. Plant Biotechnology Journal 8 , 887-899 (2010).

56. Xie, F. L. et al. Computational identification of novel microRNAs and targets in Brassica napus. FEBS Letters581 , 1464-1474 (2007).

57. Zhou, Z. S., Huang, S. Q. \& Yang, Z. M. Bioinformatic identification and expression analysis of new microRNAs from Medicago truncatula. Biochemical and Biophysical Research Communications374, 538$542(2008)$.

58. Cong, W. et al. Transgenerational memory of gene expression changes induced by heavy metal stress in rice (Oryza sativa L.).BMC Plant Biology 19, 282 (2019).

59. Ou, X. et al. Transgenerational Inheritance of Modified DNA Methylation Patterns and Enhanced Tolerance Induced by Heavy Metal Stress in Rice (Oryza sativa L.). PLOS ONE 7 , e41143 (2012).

60. Rahavi, M. R., Migicovsky, Z., Titov, V. \& Kovalchuk, I. Transgenerational Adaptation to Heavy Metal Salts in Arabidopsis.Front Plant Sci 2 , (2011).

61. Shaw, J. Heavy Metal Tolerance in Plants: Evolutionary Aspects . (CRC Press, 1989).

62. Elvira, N. J., Medina, N. G., Leo, M., Cala, V. \& Estébanez, B. Copper Content and Resistance Mechanisms in the Terrestrial Moss Ptychostomum capillare: A Case Study in an Abandoned Copper Mine in Central Spain. Arch Environ Contam Toxicol 79 , 49-59 (2020).

63. Konno, H., Nakashima, S. \& Katoh, K. Metal-tolerant moss Scopelophila cataractae accumulates copper in the cell wall pectin of the protonema. Journal of Plant Physiology 167 , 358-364 (2010).

64. Krzesłowska, M., Lenartowska, M., Mellerowicz, E. J., Samardakiewicz, S. \& Woźny, A. Pectinous cell wall thickenings formation - A response of moss protonemata cells to lead.Environmental and Experimental Botany 65 , 119-131 (2009).

65. Krzesłowska, M. \& Woźny, A. Lead uptake, localization and changes in cell ultrastructure ofFunaria hygrometrica protonemata.Biologia Plantarum (2008) doi:10.1007/BF02873855.

66. Lang, I. \& Wernitznig, S. Sequestration at the cell wall and plasma membrane facilitates zinc tolerance in the moss Pohlia drummondii.Environmental and Experimental Botany 74 , 186-193 (2011). 
67. Antreich, S., Sassmann, S. \& Lang, I. Limited accumulation of copper in heavy metal adapted mosses. Plant Physiology and Biochemistry 101, 141-148 (2016).

68. Boquete, M. T., Lang, I., Weidinger, M., Richards, C. L. \& Alonso, C. Patterns and mechanisms of heavy metal accumulation and tolerance in two terrestrial moss species with contrasting habitat specialization.Environmental and Experimental Botany 182, 104336 (2021).

69. Sabovljević, M. S. et al. Metal accumulation in the acrocarp moss Atrichum undulatum under controlled conditions. Environmental Pollution 256, 113397 (2020).

70. Bellini, E. et al. The Moss Leptodictyum riparium Counteracts Severe Cadmium Stress by Activation of Glutathione Transferase and Phytochelatin Synthase, but Slightly by Phytochelatins. Int J Mol Sci 21 , 1583 (2020).

71. van Gurp, T. P. et al. epiGBS: reference-free reduced representation bisulfite sequencing. Nat Methods 13, 322-324 (2016).

72. Shaw, A. J. Population Biology of the Rare Copper Moss, Scopelophila cataractae. American Journal of Botany 80 , 1034-1041 (1993).

73. Simms, E. L. Defining tolerance as a norm of reaction.Evolutionary Ecology 14, 563-570 (2000).

74. Mounger, J. et al. Inheritance of DNA methylation differences in the mangrove Rhizophora mangle. Evolution 85 Development 23 , 351-374 (2021).

75. Bolger, A. M., Lohse, M. \& Usadel, B. Trimmomatic: a flexible trimmer for Illumina sequence data. Bioinformatics 30 , 2114-2120 (2014).

76. Grabherr, M. G. et al. Full-length transcriptome assembly from RNA-Seq data without a reference genome. Nat Biotechnol29 , 644-652 (2011).

77. Haas, B. J. et al. De novo transcript sequence reconstruction from RNA-seq using the Trinity platform for reference generation and analysis. Nat Protoc 8, 1494-1512 (2013).

78. Fu, L., Niu, B., Zhu, Z., Wu, S. \& Li, W. CD-HIT: accelerated for clustering the next-generation sequencing data. Bioinformatics 28, 3150-3152 (2012).

79. Langmead, B. \& Salzberg, S. L. Fast gapped-read alignment with Bowtie 2. Nat Methods 9, 357-359 (2012).

80. Simão, F. A., Waterhouse, R. M., Ioannidis, P., Kriventseva, E. V. \& Zdobnov, E. M. BUSCO: assessing genome assembly and annotation completeness with single-copy orthologs. Bioinformatics 31 , 3210-3212 (2015).

81. Li, B. \& Dewey, C. N. RSEM: accurate transcript quantification from RNA-Seq data with or without a reference genome. BMC Bioinformatics 12, 323 (2011).

82. Götz, S. et al. High-throughput functional annotation and data mining with the Blast2GO suite. Nucleic Acids Research 36 , 3420-3435 (2008).

83. OmicsBox - Bioinformatics Made Easy, BioBam Bioinformatics, March 3, 2019, . (2019).

84. Conesa, A. et al. Blast2GO: a universal tool for annotation, visualization and analysis in functional genomics research.Bioinformatics 21 , 3674-3676 (2005).

85. Huerta-Cepas, J. et al. eggNOG 5.0: a hierarchical, functionally and phylogenetically annotated orthology resource based on 5090 organisms and 2502 viruses. Nucleic Acids Research47, D309-D314 (2019).

86. Smit, A., Hubley, R, \& Green, P. RepeatMasker Open-4.0.(2013). 
87. Buchfink, B., Xie, C. \& Huson, D. H. Fast and sensitive protein alignment using DIAMOND. Nat Methods 12, 59-60 (2015).

88. R Core Team. R: A language and environment for statistical computing. $R$ Foundation for Statistical Computing, Vienna, Austria. (2018).

89. RStudio Team. RStudio: Integrated Development for R. RStudio, PBC, Boston, MA . (2019).

90. Kamvar, Z. N., Tabima, J. F. \& Grünwald, N. J. Poppr: an R package for genetic analysis of populations with clonal, partially clonal, and/or sexual reproduction. PeerJ 2 , e281 (2014).

91. Jombart, T. adegenet: a R package for the multivariate analysis of genetic markers. Bioinformatics $\mathbf{2 4}$, 1403-1405 (2008).

92. Dray, S. \& Dufour, A.-B. The ade4 Package: Implementing the Duality Diagram for Ecologists. Journal of Statistical Software 22, 1-20 (2007).

93. Goudet, J. hierfstat, a package for $\mathrm{r}$ to compute and test hierarchical F-statistics. Molecular Ecology Notes 5 , 184-186 (2005).

94. Legendre, P. \& Anderson, M. J. Distance-Based Redundancy Analysis: Testing Multispecies Responses in Multifactorial Ecological Experiments.Ecological Monographs 69 , 1-24 (1999).

95. Oksanen, J. et al. vegan: Community Ecology Package . (2020).

96. Wang, Y., Qian, M., Ruan, P., Teschendorff, A. E. \& Wang, S. Detection of epigenetic field defects using a weighted epigenetic distance-based method. Nucleic Acids Res 47 , e6-e6 (2019).

97. Feng, H., Conneely, K. N. \& Wu, H. A Bayesian hierarchical model to detect differentially methylated loci from single nucleotide resolution sequencing data. Nucleic Acids Res 42 , e69 (2014).

98. Park, Y. \& Wu, H. Differential methylation analysis for BS-seq data under general experimental design. Bioinformatics 32 , 1446-1453 (2016).

99. Zheng, X., Zhang, N., Wu, H.-J. \& Wu, H. Estimating and accounting for tumor purity in the analysis of DNA methylation data from cancer studies. Genome Biology 18, 17 (2017).

100. Robinson, M. D., McCarthy, D. J. \& Smyth, G. K. edgeR: a Bioconductor package for differential expression analysis of digital gene expression data. Bioinformatics 26 , 139-140 (2010).

101. Al-Shahrour, F., Díaz-Uriarte, R. \& Dopazo, J. FatiGO: a web tool for finding significant associations of Gene Ontology terms with groups of genes. Bioinformatics 20 , 578-580 (2004).

102. Kent, W. J. BLAT-The BLAST-Like Alignment Tool. Genome Res. 12 ，656-664 (2002).

103. Shaw, A. J. Genetic biogeography of the rate 'copper moss', Scopelophila cataractae (Pottiaceae). Plant Systematics and Evolution 197, 43-58 (1995).

104. Shaw, J. Evolution of Heavy Metal Tolerance in Bryophytes. II. An Ecological and Experimental Investigation of the 'Copper Moss,' Scopelophila cataractae (Pottiaceae). American Journal of Botany $\mathbf{7 4}$, 813-821 (1987).

105. Nomura, T. \& Hasezawa, S. Regulation of gemma formation in the copper moss Scopelophila cataractae by environmental copper concentrations. J Plant Res 124, 631-638 (2011).

106. Shaw, A. J. Morphological Uniformity Among Widely Disjunct Populations of the Rare 'Copper Moss,' Scopelophila cataractae (Pottiaceae). Systematic Botany 18, 525-537 (1993).

107. Herrera, C. M. \& Bazaga, P. Epigenetic correlates of plant phenotypic plasticity: DNA methylation differs between prickly and nonprickly leaves in heterophyllous Ilex aquifolium (Aquifoliaceae) trees. Botanical Journal of the Linnean Society 171 , 441-452 (2013). 
108. Róis, A. S. et al. Epigenetic rather than genetic factors may explain phenotypic divergence between coastal populations of diploid and tetraploid Limonium spp. (Plumbaginaceae) in Portugal. BMC Plant Biol 13,205 (2013).

109. Alonso, C., Pérez, R., Bazaga, P., Medrano, M. \& Herrera, C. M. Individual variation in size and fecundity is correlated with differences in global DNA cytosine methylation in the perennial herb Helleborus foetidus (Ranunculaceae). American Journal of Botany101 , 1309-1313 (2014).

110. Medrano, M., Herrera, C. M. \& Bazaga, P. Epigenetic variation predicts regional and local intraspecific functional diversity in a perennial herb. Molecular Ecology 23 , 4926-4938 (2014).

111. Platt, A., Gugger, P. F., Pellegrini, M. \& Sork, V. L. Genome-wide signature of local adaptation linked to variable CpG methylation in oak populations. Molecular Ecology 24, 3823-3830 (2015).

112. Richards, C. L. \& Pigliucci, M. Epigenetic Inheritance. A Decade into the Extended Evolutionary Synthesis. $P G(2020)$ doi:10.30460/99624.

113. Puy, J. et al. Competition-induced transgenerational plasticity influences competitive interactions and leaf decomposition of offspring. New Phytologist 229 , 3497-3507 (2021).

114. Yadav, N. S. et al. Multigenerational exposure to heat stress induces phenotypic resilience, and genetic and epigenetic variations in Arabidopsis thaliana offspring . 2020.11.30.405365 https://www.biorxiv.org/content/10.1101/2020.11.30.405365v1 (2020) doi:10.1101/2020.11.30.405365.

115. Verhoeven, K. J. F., Jansen, J. J., van Dijk, P. J. \& Biere, A. Stress-induced DNA methylation changes and their heritability in asexual dandelions. New Phytologist 185, 1108-1118 (2010).

116. Boquete, M. T., Muyle, A. \& Alonso, C. Plant epigenetics: phenotypic and functional diversity beyond the DNA sequence.American Journal of Botany 108, 553-558 (2021).

117. Lang, D. et al. The Physcomitrella patens chromosome-scale assembly reveals moss genome structure and evolution. The Plant Journal 93 , 515-533 (2018).

118. Diop, S. I. et al. A pseudomolecule-scale genome assembly of the liverwort Marchantia polymorpha. The Plant Journal101 , 1378-1396 (2020).

119. Schmid, M. W. et al. Extensive epigenetic reprogramming during the life cycle of Marchantia polymorpha. Genome Biology 19 , 9 (2018).

120. Zemach, A., McDaniel, I. E., Silva, P. \& Zilberman, D. Genome-Wide Evolutionary Analysis of Eukaryotic DNA Methylation. Science(2010).

121. Alvarez, M. et al. Reduced representation characterization of genetic and epigenetic differentiation to oil pollution in the foundation plant Spartina alterniflora . 426569 https://www.biorxiv.org/content/10.1101/426569v3 (2020) doi:10.1101/426569.

122. Robertson, M. et al. Combining epiGBS markers with long read transcriptome sequencing to assess differentiation associated with habitat in Reynoutria (aka Fallopia) . 2020.09.30.317966 https://www.biorxiv.org/content/10.1101/2020.09.30.317966v1 (2020) doi:10.1101/2020.09.30.317966.

123. Hasan, M. K. et al. Responses of Plant Proteins to Heavy Metal Stress-A Review. Front. Plant Sci. 8 , (2017).

124. Lemire, J. A., Harrison, J. J. \& Turner, R. J. Antimicrobial activity of metals: mechanisms, molecular targets and applications. Nat Rev Microbiol 11 , 371-384 (2013).

125. Tamás, M. J., Sharma, S. K., Ibstedt, S., Jacobson, T. \& Christen, P. Heavy Metals and Metalloids As a Cause for Protein Misfolding and Aggregation. Biomolecules 4 , 252-267 (2014). 
126. Choudhary, S. P., Kanwar, M., Bhardwaj, R., Yu, J.-Q. \& Tran, L.-S. P. Chromium Stress Mitigation by Polyamine-Brassinosteroid Application Involves Phytohormonal and Physiological Strategies in Raphanus sativus L. PLOS ONE 7 , e33210 (2012).

127. Hussain, A., Nazir, F. \& Fariduddin, Q. Polyamines (spermidine and putrescine) mitigate the adverse effects of manganese induced toxicity through improved antioxidant system and photosynthetic attributes in Brassica juncea. Chemosphere 236 , 124830 (2019).

128. Nahar, K., Hasanuzzaman, M., Suzuki, T. \& Fujita, M. Polyamines-induced aluminum tolerance in mung bean: A study on antioxidant defense and methylglyoxal detoxification systems.Ecotoxicology 26 , $58-73$ (2017).

129. Wang, X., Shi, G., Xu, Q. \& Hu, J. Exogenous polyamines enhance copper tolerance of Nymphoides peltatum. Journal of Plant Physiology 164, 1062-1070 (2007).

130. Sarry, J.-E. et al. The early responses of Arabidopsis thaliana cells to cadmium exposure explored by protein and metabolite profiling analyses. PROTEOMICS 6 , 2180-2198 (2006).

Table 1: Average DNA methylation (Mean) and standard deviation of DNA methylation (Std. Dev.) per group (i.e. each unique population - Pop. - and treatment - Treat. - combination) for each separate sequence context (CG, CHG, CHH), and across all contexts (all) in samples of the copper moss Scopelophila cataractae obtained with the complete single methylation polymorphism matrix (All SMPs; n=43,365 SMPs), and the ccomplete and polymorphic matrix (Polym. SMPs; n=3,769 SMPs).

\begin{tabular}{|c|c|c|c|c|c|c|c|c|c|c|}
\hline Pop & Treat & Dataset & CG & CG & CHG & CHG & $\mathrm{CHH}$ & $\mathrm{CHH}$ & all & all \\
\hline \multirow{7}{*}{ Sc1 } & & \multirow{4}{*}{ All SMPs } & Mean & Std. Dev. & Mean & Std. Dev. & Mean & Std. Dev. & Mean & Std. Dev. \\
\hline & $\mathrm{C}$ & & 0.63 & 2.33 & 11.98 & 31.49 & 0.66 & 2.34 & 3.25 & 15.95 \\
\hline & $\mathrm{Cd}$ & & 0.64 & 3.36 & 12.06 & 31.64 & 0.67 & 3.17 & 3.28 & 16.15 \\
\hline & $\mathrm{Cu}$ & & 0.74 & 3.04 & 12.07 & 31.51 & 0.81 & 2.83 & 3.38 & 16.03 \\
\hline & $\mathrm{C}$ & \multirow[t]{3}{*}{ Polim. SMPs } & 2.38 & 4.98 & 34.39 & 45.38 & 2.29 & 4.22 & 10.67 & 27.37 \\
\hline & $\mathrm{Cd}$ & & 2.75 & 7.70 & 34.61 & 45.66 & 2.29 & 5.94 & 10.81 & 27.81 \\
\hline & $\mathrm{Cu}$ & & 2.96 & 7.16 & 34.45 & 45.27 & 2.85 & 5.00 & 11.10 & 27.38 \\
\hline \multirow[t]{6}{*}{$\mathrm{Sc} 2$} & $\mathrm{C}$ & \multirow[t]{3}{*}{ All SMPs } & 0.70 & 3.64 & 12.09 & 31.64 & 0.69 & 3.36 & 3.31 & 16.19 \\
\hline & $\mathrm{Cd}$ & & 0.76 & 3.27 & 12.15 & 31.55 & 0.80 & 2.99 & 3.40 & 16.08 \\
\hline & $\mathrm{Cu}$ & & 0.73 & 2.86 & 12.09 & 31.52 & 0.71 & 2.54 & 3.33 & 16.01 \\
\hline & $\mathrm{C}$ & \multirow[t]{3}{*}{ Polim. SMPs } & 2.87 & 8.10 & 34.89 & 45.48 & 2.51 & 6.30 & 11.02 & 27.82 \\
\hline & $\mathrm{Cd}$ & & 3.06 & 7.70 & 35.00 & 45.23 & 2.60 & 5.18 & 11.14 & 27.57 \\
\hline & $\mathrm{Cu}$ & & 3.21 & 7.05 & 34.43 & 45.49 & 2.33 & 4.81 & 10.88 & 27.51 \\
\hline \multirow[t]{6}{*}{ Sc3 } & $\mathrm{C}$ & \multirow[t]{3}{*}{ All SMPs } & 0.74 & 3.69 & 12.05 & 31.51 & 0.78 & 3.53 & 3.36 & 16.14 \\
\hline & $\mathrm{Cd}$ & & 0.66 & 3.04 & 12.04 & 31.52 & 0.72 & 3.03 & 3.30 & 16.06 \\
\hline & $\mathrm{Cu}$ & & 0.66 & 2.64 & 12.05 & 31.53 & 0.74 & 2.52 & 3.32 & 16.00 \\
\hline & $\mathrm{C}$ & \multirow[t]{3}{*}{ Polim. SMPs } & 3.17 & 8.53 & 34.61 & 44.98 & 2.75 & 6.74 & 11.14 & 27.57 \\
\hline & $\mathrm{Cd}$ & & 2.72 & 7.50 & 34.42 & 45.44 & 2.60 & 5.89 & 10.92 & 27.61 \\
\hline & $\mathrm{Cu}$ & & 2.89 & 7.08 & 34.73 & 45.25 & 2.77 & 5.43 & 11.12 & 27.48 \\
\hline \multirow[t]{6}{*}{ Sc4 } & $\mathrm{C}$ & \multirow[t]{3}{*}{ All SMPs } & 0.64 & 3.01 & 11.98 & 31.52 & 0.59 & 2.44 & 3.22 & 16.01 \\
\hline & $\mathrm{Cd}$ & & 0.75 & 2.80 & 12.11 & 31.50 & 0.72 & 2.41 & 3.34 & 15.99 \\
\hline & $\mathrm{Cu}$ & & 0.61 & 3.06 & 12.00 & 31.58 & 0.62 & 2.94 & 3.23 & 16.08 \\
\hline & $\mathrm{C}$ & \multirow[t]{3}{*}{ Polim. SMPs } & 2.44 & 7.34 & 34.24 & 45.23 & 1.71 & 4.05 & 10.34 & 27.46 \\
\hline & $\mathrm{Cd}$ & & 2.88 & 6.79 & 34.68 & 45.27 & 2.22 & 4.08 & 10.81 & 27.44 \\
\hline & $\mathrm{Cu}$ & & 2.62 & 6.81 & 34.33 & 45.59 & 2.08 & 5.20 & 10.59 & 27.63 \\
\hline
\end{tabular}

Table 2: Results of the distance-based redundancy analysis (dbRDA) carried out to test the effect of population, treatment, and their interaction on genome wide DNA methylation for each of the common garden 
experiments (Overall model). The results of the permutation test $(\mathrm{n}=9999)$ for the significance of each of the predictors are also shown (Predictors). $d_{\mathrm{st}}$ : pairwise distance matrix calculated with the methylation level and variance of each cytosine in each sample. CG-Cd: common garden comparing control and Cdtreated plants; CG-Cu: common garden comparing control and Cu-treated plants; Df: degrees of freedom; adj. $R^{2}$ : adjusted $R^{2}$.

\begin{tabular}{lllllllll}
\hline Experiment & Response & Model & Predictor & Df & Variance & $\mathrm{F}$ & $\operatorname{Pr}(>\mathrm{F})$ & adj. $\mathrm{R}^{2}$ \\
\hline CG-Cd & $d_{\text {st }}$ & Overall model & Model & 7 & 11405 & 5.08 & 0.0001 & 61.3 \\
& & & Residual & 11 & 3531 & & & \\
& & \multirow{2}{*}{ Predictors } & Population & 3 & 4916 & 5.11 & 0.0001 & 21.6 \\
& & Treatment & 1 & 1685 & 5.25 & 0.0001 & 8.8 \\
& & Pop.:Treat. & 3 & 4803 & 4.99 & 0.0001 & 33.1 \\
$\mathrm{CG}-\mathrm{Cu}$ & \multirow{2}{*}{$d_{\text {st }}$} & \multirow{2}{*}{ Overall model } & Residual & 11 & 3531 & & & \\
& & & Model & 7 & 10859 & 4.48 & 0.0001 & 56.2 \\
& & Residual & 12 & 4152 & & & \\
& & \multirow{2}{*}{ Predictors } & Population & 3 & 4881 & 4.70 & 0.0001 & 21.3 \\
& & Treatment & 1 & 1456 & 4.21 & 0.0001 & 7.0 \\
& & & Pop.:Treat. & 3 & 4522 & 4.36 & 0.0001 & 29.4 \\
& & & Residual & 12 & 4152 & & & \\
\hline
\end{tabular}

\section{Figure captions}

Figure 1: Left panel : ordination plots of the distance-based redundancy analysis (dbRDA) on the epigenetic distances between cadmium-treated and control plants (Cd vs. C; A1), and between copper-treated and control plants ( $\mathrm{Cu}$ vs. C; A2) from four populations of the copper moss Scopelophila cataractae $(\mathrm{n}=2-3$ replicates per population and treatment). RDA1: first constrained ordination axis explaining 20.6 (A1) and 15.2 (A2) of the total variance of the data; RDA2: second constrained ordination axis explaining 14.3 (A1) and 13.3 (A2) of the total variance of the data. Asterisks mark overlapping observations from the same group. Central panel : difference in the methylation level (i.e. methylation change) of differentially methylated cytosine positions (DMPs; fdr $<0.05)$ between cadmium-treated and control plants $(\mathrm{Cd}$ vs. C; B1), and between copper-treated and control plants ( $\mathrm{Cu}$ vs. C; B2) from four populations of the copper moss Scopelophila cataractae (Sc1 to Sc4); values at the bottom of each graph indicate the number of DMPs for each contrast (-: up-methylated; —: down-methylated); red lines delimit the area in which absolute methylation differences are lower than 10.Right panel : multidimensional scaling (MDS) plot of the gene expression profiles in control and $\mathrm{Cu}$-treated samples from two populations of the copper moss Scopelophila cataractae (Sc3 and Sc4; C1). Heatmap of the normalized expression levels (i.e. Row z-scores) of the differentially expressed transcripts in response to $\mathrm{Cu}$ shared between $\mathrm{Sc} 3$ and $\mathrm{Sc} 4$ (C2).

Figure 2: Top panel : word cloud representation of gene ontology (GO) terms related to all molecular functions significantly over-represented in the list of differentially expressed transcripts (DETs) in response to $\mathrm{Cu}$ in Sc3 (A; $\mathrm{n}=22 \mathrm{GOs})$ and $\mathrm{Sc} 4(\mathrm{~B} ; \mathrm{n}=4 \mathrm{GOs})$. The size of the words is proportional to the frequency of the GO term in the DETs list. GOs inside grey rectangles show under-represented terms in the DETs' list. Bottom panel : circular plots of the functional categories created by grouping downregulated transcripts of Sc3 (C) and Sc4 (D) in response to $\mathrm{Cu}$ according to their annotation descriptions. The percent of transcripts within the group with respect to the total number of DETs is shown between parentheses. N: total number of DETs included in this classification for each population. 

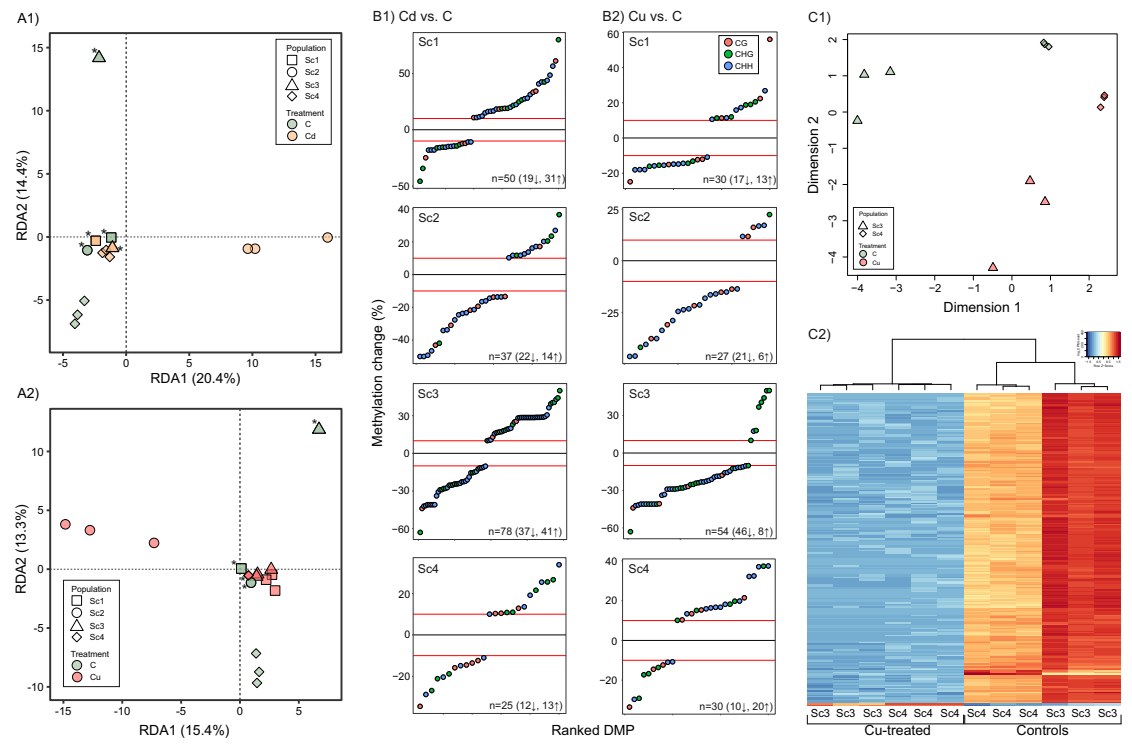


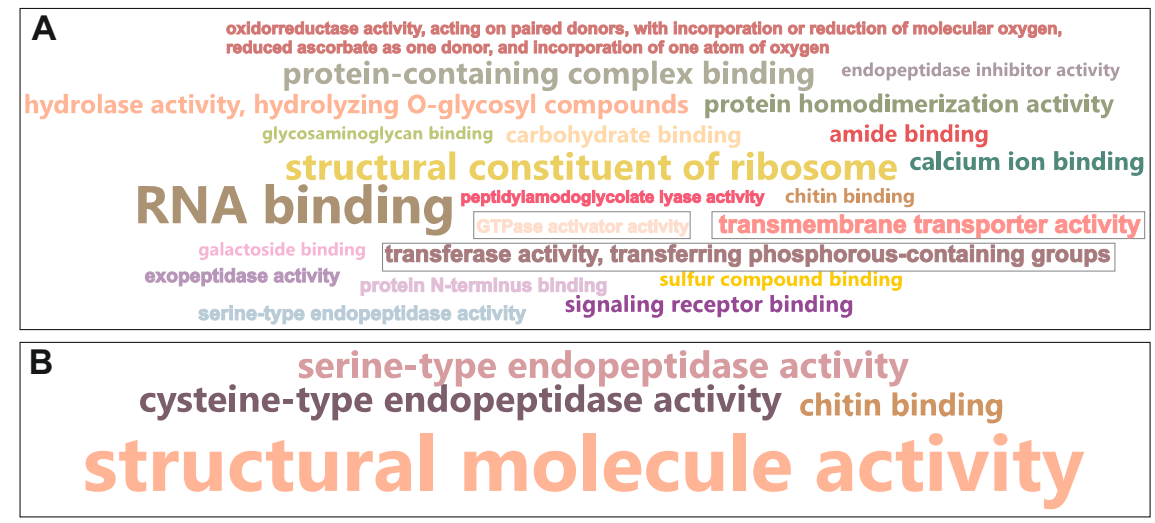
C 1. Protein \& ribosome biosynthesis (8.8\%)
2. Protein degradation \& turnover $(4.3 \%)$
3. Redox activity $(2.5 \%)$
4. Protein repair (proper folding) $(1.9 \%)$
5. Ca homeostasis \& Ca-, non Ca-signaling (1.6\%)
6. Energy metabolism (1.6\%)
7. Membrane transporters \& proteins associated $(1.5 \%)$
8. RNA metabolism (biosynthesis \& degradation) (1.2\%)
9. Vesicle trafficking $(0.6 \%)$
10. Fatty acid oxidation $(0.4 \%)$
11. Chromatin organization $(0.4 \%)$
12. Polyamine biosynthesis \& degradation $(0.4 \%)$
13. Purine metabolism (0.3\%)

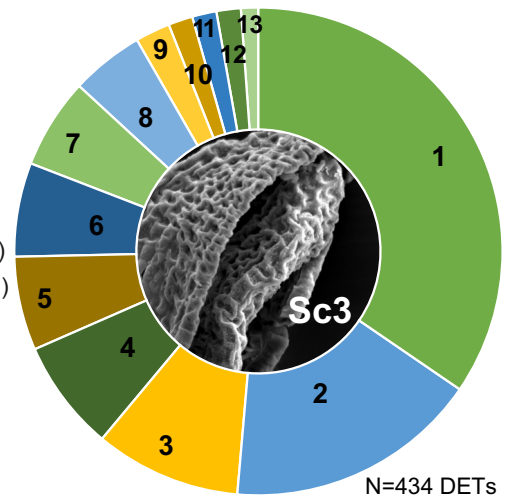

D

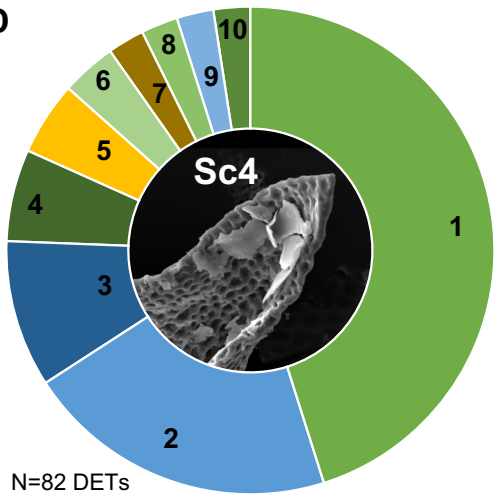

1. Protein \& ribosome biosynthesis $(14.3 \%)$

2. Protein degradation \& turnover $(6.6 \%)$

3. Energy metabolism (3.1\%)

4. Protein repair (proper folding) (1.9\%)

5. Redox activity $(1.6 \%)$

6. Purine metabolism (1.2\%)

7. Ca homeostasis \& Ca-, non Ca-signaling $(0.8 \%)$

8. Membrane transporters \& proteins associated $(0.8 \%)$

9. RNA metabolism (biosynthesis \& degradation) $(0.8 \%)$

10. Polyamine biosynthesis \& degradation $(0.8 \%)$

\section{Hosted file}

Table1.docx available at https://authorea.com/users/460231/articles/556279-molecularbasis-of-intraspecific-differentiation-for-heavy-metal-tolerance-in-the-copper-mossscopelophila-cataractae

\section{Hosted file}

Table2.docx available at https://authorea.com/users/460231/articles/556279-molecularbasis-of-intraspecific-differentiation-for-heavy-metal-tolerance-in-the-copper-mossscopelophila-cataractae 